\title{
Immune networks: multi-tasking capabilities at medium load
}

\author{
E Agliari ${ }^{1,2}$, A Annibale ${ }^{3,4}$, A Barra ${ }^{5}$, A C C Coolen ${ }^{4,6}$ and D Tantari ${ }^{7}$ \\ ${ }^{1}$ Dipartimento di Fisica, Università degli Studi di Parma, Viale GP Usberti 7/A, I-43124 Parma, \\ Italy \\ 2 INFN, Gruppo Collegato di Parma, Viale Parco Area delle Scienze 7/A, I-43100 Parma, Italy \\ ${ }^{3}$ Department of Mathematics, King's College London, The Strand, London WC2R 2LS, UK \\ ${ }^{4}$ Institute for Mathematical and Molecular Biomedicine, King's College London, \\ Hodgkin Building, London SE1 1UL, UK \\ ${ }^{5}$ Dipartimento di Fisica, Sapienza Università di Roma, P.le Aldo Moro 2, I-00185 Roma, Italy \\ ${ }^{6}$ London Institute for Mathematical Sciences, 35a South St, Mayfair, London W1K 2XF, UK \\ 7 Dipartimento di Matematica, Sapienza Università di Roma, P.le Aldo Moro 2, I-00185 Roma, \\ Italy \\ E-mail: agliari@fis.unipr.it, alessia.annibale@kcl.ac.uk, adriano.barra@roma1.infn.it, \\ ton.coolen@kcl.ac.uk and tantari@mat.uniroma1.it
}

Received 28 February 2013, in final form 8 July 2013

Published 29 July 2013

Online at stacks.iop.org/JPhysA/46/335101

\begin{abstract}
Associative network models featuring multi-tasking properties have been introduced recently and studied in the low-load regime, where the number $P$ of simultaneously retrievable patterns scales with the number $N$ of nodes as $P \sim \log N$. In addition to their relevance in artificial intelligence, these models are increasingly important in immunology, where stored patterns represent strategies to fight pathogens and nodes represent lymphocyte clones. They allow us to understand the crucial ability of the immune system to respond simultaneously to multiple distinct antigen invasions. Here we develop further the statistical mechanical analysis of such systems, by studying the mediumload regime, $P \sim N^{\delta}$ with $\delta \in(0,1]$. We derive three main results. First, we reveal the nontrivial architecture of these networks: they exhibit a high degree of modularity and clustering, which is linked to their retrieval abilities. Second, by solving the model we demonstrate for $\delta<1$ the existence of large regions in the phase diagram where the network can retrieve all stored patterns simultaneously. Finally, in the high-load regime $\delta=1$ we find that the system behaves as a spin-glass, suggesting that finite-connectivity frameworks are required to achieve effective retrieval.
\end{abstract}

PACS numbers: 75.10.Nr, 87.18.Vf

(Some figures may appear in colour only in the online journal) 


\section{Introduction}

After a pioneering paper [1] followed by a long period of dormancy, recent years have witnessed a surge of interest in statistical mechanical models of the immune system [2-10]. This description complements the more standard approaches, which tend to be phrased in the language of dynamical systems [11-14], network theory [15-20] or multi-scale mathematical biology [21-23].

In addition to offering an alternative, complementary, formal perspective on lymphocyte dynamics, one of the main advantages of statistical mechanics approaches is that they reveal collective functionality of the constituent elements of large systems to be an emergent property of the network via which they interact. This fruitful perspective originated in theoretical physics, but is appreciated also by pure immunologists; see e.g. [24, 25]. In particular, the problem of parallel processing as tackled in this work, i.e. understanding the natural ability of the immune system to deal with several pathogens at the same time, is becoming a crucial point in modern immunology $[26,27]$. The long and rich history of statistical mechanical modelling of neural networks, which share several important features with immune networks [28, 29], enables us to transfer quantitative technology and intuition from neural network modelling to the study of signalling in the immune system. However, the price to pay for this progress is having to start with a symmetric theory, i.e. an equilibrium picture.

There is indeed an intriguing and fruitful analogy (from a modelling perspective) between neural networks, which have been modelled in statistical mechanics quite extensively, and immune networks. Let us highlight the similarities and differences. In neural networks the nodes represent neurons, which interact with each other directly through Hebbian synaptic couplings. In (adaptive) immune systems, effector branches (B-clones) and coordinator branches (helper and suppressor T-clones), interact via signalling proteins called cytokines. The latter can represent both eliciting and suppressive signals. Neural and immune systems are both able to learn (e.g. how to fight new antigens), memorize (e.g. previously encountered antigens) and 'think' (e.g. select the best strategy to cope with pathogens). However, neural networks are designed for serial processing: neurons perform collectively to retrieve a single pattern at a time. This is not acceptable in the immune context. Multiple antigens will normally be present at the same time, which requires the simultaneous recall of multiple patterns (i.e. of multiple defense strategies). Moreover, the architectures of neural and immune networks are very different. A model with fully connected topology, mathematically convenient but without a basis in biological reality, is tolerable for neural networks where each neuron is known to have a huge number of connections with others. In contrast, in immune networks, where interactions among lymphocytes are much more specific, the underlying topology must be carefully modelled and is expected to play a crucial operational role. From a theoretical physics perspective, a network of interacting B- and T-cells resembles a bipartite spin-glass. It was recently shown that such bipartite spin-glasses exhibit retrieval features which are deeply related to their structures [30,31], and this can be summarized as follows.

- There exists a structural equivalence between Hopfield neural networks and bipartite spinglasses. In particular, the two systems share the same partition function, and hence the same thermodynamics $[32,33]$.

- One can either dilute directly a Hopfield network, or its underlying bipartite spin-glass. The former does not affect pattern retrieval qualitatively [34-39], whereas the latter causes a switch from serial to parallel processing [30,31] (i.e. to simultaneous pattern recall).

- Simultaneous pattern recall is essential in the context of immunology, since it implies the ability to respond to multiple antigens simultaneously. The analysis of such systems requires a combination of techniques from statistical mechanics and graph theory. 
The last point is the focus of the present paper, which is organized as follows. In section 2 we describe a minimal biological scenario for the immune system, based on the analogy with neural networks. We define our model and its scaling regimes, and prepare the stage for calculations. Section 3 gives a comprehensive analysis of the topological properties of the network in the extremely diluted regime, which is the scaling regime assumed throughout our paper. Section 4 is dedicated to the statistical mechanical analysis of the system in the medium-load regime, focusing on simultaneous pattern recall of the network. Section 5 deals with the high-load regime. Here the network is found to behave as a spin-glass, suggesting that a higher degree of dilution should be implemented —in remarkable agreement with immunological findings [40, 41] —and this will be the focus of future research. The final section gives a summary of our main conclusions.

\section{Statistical mechanical modelling of the adaptive immune system}

\subsection{The underlying biology}

All mammals have an innate (broad range) immunity, managed by macrophages, neutrophils, etc, and an adaptive immune response. The latter is highly specific for particular targets, handled by lymphocytes, and the focus of this paper. To be concise, the following introduction to the adaptive immune system has already been filtered by a theoretical physics perspective, and immunological observables are expressed in 'physical' language. We refer to the excellent books $[40,41]$ for comprehensive reviews of the immune system, and to a selection of papers $[2-4,30,31,42]$ for explanations of the link between 'physical' models and biological reality. Our prime interest is in B-cells and in T-cells; in particular, among T-cells, in the subgroups of so-called 'helpers' and 'suppressors'. B-cells produce antibodies and present them on their external surface in such a way that they are able to recognize and bind pathogenic peptides. All B-cells that produce the same antibody belong to the same clone, and the ensemble of all the different clones forms the immune repertoire. This repertoire is of size $\mathcal{O}\left(10^{8}-10^{9}\right)$ clones in humans. The size of a clone, i.e. the number of identical B-cells, may vary strongly. A clone at rest may contain some $\mathcal{O}\left(10^{3}-10^{4}\right)$ cells, but when it undergoes clonal expansion its size may increase by several orders of magnitude, to up to $\mathcal{O}\left(10^{6}-10^{7}\right)$. Beyond this size the state of the immune system would be pathological, and is referred to as lymphocytosis.

When an antigen enters the body, several antibodies (i.e. several B-cells belonging to different clones) may be able to bind to it, making it chemically inert and biologically inoffensive. In this case, conditional on authorization by T-helpers (mediated via cytokines, interleukin in the example of figure 1), the binding clones undergo clonal expansion. This means that their cells start duplicating, and releasing high quantities of soluble antibodies to inhibit the enemy. After the antigen has been deleted, B-cells are instructed by T-suppressors, again via cytokines, to stop producing antibodies and undergo apoptosis. In this way the clones reduce their sizes, and order is restored. Thus, two signals are required for B-cells to start clonal expansion: the first signal is binding to antigen, the second is a 'consensus' signal, in the form of an eliciting cytokine secreted by T-helpers. This latter mechanism prevents abnormal reactions, such as autoimmune manifestations 8 .

T-helpers and T-suppressors are lymphocytes that work 'behind the scenes', regulating the immune response by coordinating the work of effector branches, which in this paper are the B-cells. To accomplish this, they are able to secrete both stimulatory and suppressive chemical signals, the cytokines [43, 44]. If within a given (small) time interval a B-clone

8 Through a phenomenon called 'cross-linking', a B-cell can also have the ability to bind a self-peptide, and may accidentally start duplication and antibody release, which is a dangerous unwanted outcome. 


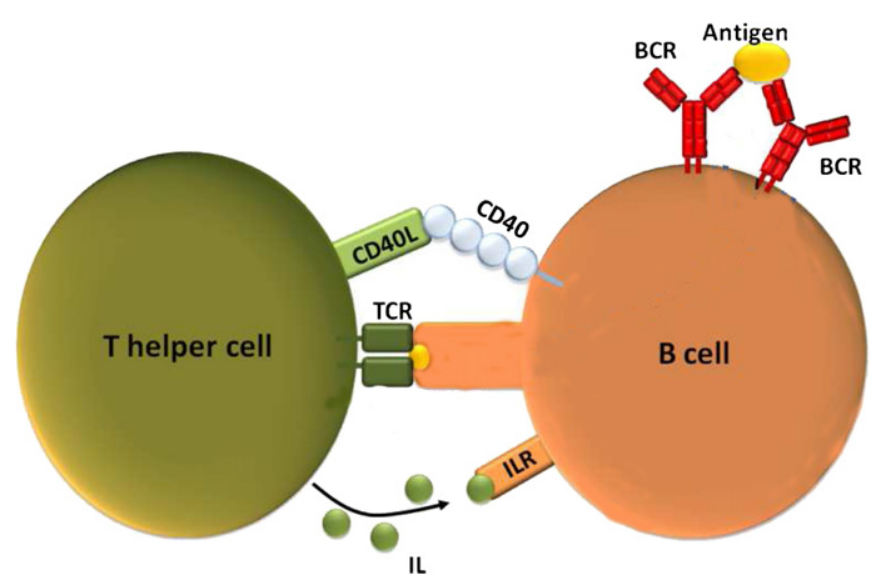

Figure 1. Schematic representation of T-cell dependent B-cell activation. The double interactions of the B-cell are shown, respectively with the antigen, via its B-cell-receptor (BCR), a $Y$-like complex, and with a T-helper (on its left). After linking the B-cell with the CD40 arm, and recognizing with its T-cell-receptor (TCR) the antigen displayed by the B-cell, the T-helper secretes interleukins (IL) which upon detection by the interleukin receptors (ILR) of the B-cell stimulate the effector functions of the B-cell. Note that the interaction via CD40 is intrinsically symmetric.

recognizes an antigen and detects an eliciting cytokine from a T-cell, it will become activated and start duplicating and secreting antibodies. This scenario is the so-called 'two-signal model' [45-48]. Conversely, when the antigen is absent and/or the cytokine signalling is suppressive, the B-cells tuned to this antigen start the apoptosis programme, and their immuno-surveillance is turned down to a rest state. For simplicity, we will from now on with the term 'helper' indicate any helper or suppressor T-cell. The focus of this study is to understand, from a statistical mechanics perspective, the ability of helpers and suppressors to coordinate and manage simultaneously a huge ensemble of B-clones (possibly all).

\subsection{A minimal model}

We consider an immune repertoire of $N_{B}$ different clones, labelled by $\mu \in\left\{1, \ldots, N_{B}\right\}$. The size of clone $\mu$ is $b_{\mu}$. In the absence of interactions with helpers, we take the clone sizes to be Gaussian distributed; without loss of generality we may take the mean to be zero and unit width, so $b_{\mu} \sim \mathcal{N}(0,1)$. A value $b_{\mu} \gg 0$ now implies that clone $\mu$ has expanded (relative to the typical clonal size), while $b_{\mu} \ll 0$ implies inhibition. The Gaussian clone size distribution is supported both by experiments and by theoretical arguments [4]. Similarly, we imagine having $N_{T}$ helper clones, labelled by $i \in\left\{1, \ldots, N_{T}\right\}$. The state of helper clone $i$ is denoted by $\sigma_{i}$. For simplicity, helpers are assumed to be in only two possible states: secreting cytokines $\left(\sigma_{i}=+1\right)$ or quiescent $\left(\sigma_{i}=-1\right)$. Clone sizes $b_{\mu}$ and the helper states $\sigma_{i}$ are dynamical variables. We will abbreviate $\sigma=\left(\sigma_{1}, \ldots, \sigma_{N_{T}}\right) \in\{-1,1\}^{N_{T}}$, and $\boldsymbol{b}=\left(b_{1}, \ldots, b_{N_{B}}\right) \in \mathbb{R}^{N_{B}}$.

The interaction between the helpers and B-clones is implemented by cytokines. These are taken to be frozen (quenched) discrete variables. The effect of a cytokine secreted by helper $i$ and detected by clone $\mu$ can be nonexistent $\left(\xi_{i}^{\mu}=0\right)$, excitatory $\left(\xi_{i}^{\mu}=1\right)$, or inhibitory $\left(\xi_{i}^{\mu}=-1\right)$. To achieve a Hamiltonian formulation of the system, and thereby enable equilibrium analysis, we have to impose symmetry of the cytokine interactions. So, in addition to the B-clones being influenced by cytokine signals from helpers, the helpers will similarly feel a signal from the B-clones. This symmetry assumption can be viewed 


$$
\text { T-cells } \quad \text {-cells }
$$
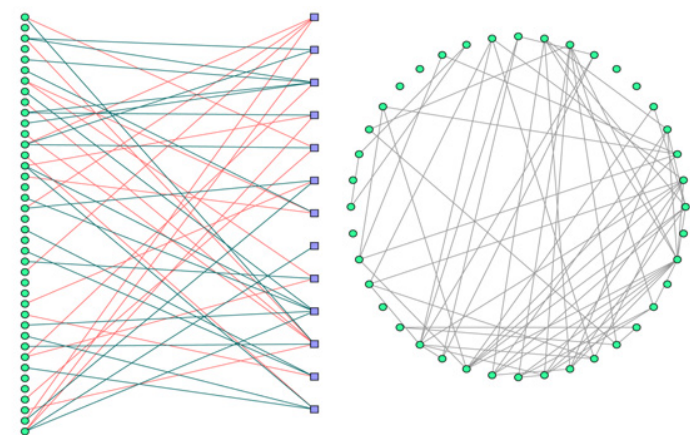

T-cells only

Figure 2. Left: schematic representation of the bipartite spin-glass which models the interaction between B- and T-cells through cytokines. The latter are drawn as coloured links, with red representing stimulatory cytokines (positive couplings) and black representing inhibiting ones (negative couplings). Note that the network is diluted. Right: the equivalent associative multitasking network consisting of T-cells only, obtained by integrating out the B-cells. This network is also diluted, with links given by the Hebbian prescription.

as a necessary first step, to be relaxed in future investigations, similar in spirit to the early formulation of symmetric spin-glass models for neural networks $[49,50]$. We are then led to a Hamiltonian $\hat{\mathcal{H}}(\boldsymbol{b}, \sigma \mid \xi)$ for the combined system of the following form (modulo trivial multiplicative factors):

$$
\hat{\mathcal{H}}(\boldsymbol{b}, \boldsymbol{\sigma} \mid \xi)=-\frac{1}{\sqrt{N_{T}}} \sum_{i=1}^{N_{T}} \sum_{\mu=1}^{N_{B}} \xi_{i}^{\mu} \sigma_{i} b_{\mu}+\frac{1}{2 \sqrt{\beta}} \sum_{\mu=1}^{N_{B}} b_{\mu}^{2} .
$$

In the language of disordered systems, this is a bipartite spin-glass. We can integrate out the variables $b_{\mu}$, and map our system to a model with helper-helper interactions only. The partition function $Z_{N_{T}}(\beta, \xi)$, at inverse clone size noise level $\sqrt{\beta}$ (which is the level consistent with our assumption $b_{\mu} \sim \mathcal{N}(0,1)$ ) follows straightforwardly, and reveals the mathematical equivalence with an associative attractor network:

$$
\begin{aligned}
Z_{N_{T}}(\beta, \xi) & =\sum_{\sigma} \int \mathrm{d} b_{1} \ldots \mathrm{d} b_{N_{B}} \exp [-\sqrt{\beta} \hat{\mathcal{H}}(\boldsymbol{b}, \boldsymbol{\sigma} \mid \xi)] \\
& =\sum_{\sigma} \exp [-\beta \mathcal{H}(\boldsymbol{\sigma} \mid \xi)],
\end{aligned}
$$

in which, modulo an irrelevant additive constant,

$$
\mathcal{H}(\boldsymbol{\sigma} \mid \xi)=-\frac{1}{2} \sum_{i j=1}^{N_{T}} \sigma_{i} J_{i j} \sigma_{j}, \quad J_{i j}=\frac{1}{N_{T}} \sum_{\mu=1}^{N_{B}} \xi_{i}^{\mu} \xi_{j}^{\mu} .
$$

Thus, the system with Hamiltonian $\hat{\mathcal{H}}(\boldsymbol{b}, \boldsymbol{\sigma} \mid \xi)$, where helpers and B-clones interact through cytokines, is thermodynamically equivalent to a Hopfield-type associative network represented by $\mathcal{H}(\sigma \mid \xi)$, in which helpers mutually interact through an effective Hebbian coupling. See figure 2. Learning a pattern in this model then means adding a new B-clone with an associated string of new cytokine variables.

If there are no zero values for the $\left\{\xi_{i}^{\mu}\right\}$, the system characterized by (3) is well known in artificial intelligence research. It is able to retrieve each of the $N_{B}$ 'patterns' $\left(\xi_{1}^{\mu}, \ldots, \xi_{N}^{\mu}\right)$, provided these patterns are sufficiently uncorrelated, and both the ratio $\alpha=N_{B} / N_{T}$ and the 
noise level $1 / \beta$ are sufficiently small $[4,35,51,56]$. Retrieval quality can be quantified by introducing $N_{B}$ suitable order parameters, viz. $m_{\mu}(\sigma)=N_{T}^{-1} \sum_{i} \xi_{i}^{\mu} \sigma_{i}$, in terms of which the new Hamiltonian (3) can be written as

$$
\mathcal{H}(\boldsymbol{\sigma} \mid \xi)=-\frac{N_{T}}{2} \sum_{\mu=1}^{N_{T}} m_{\mu}^{2}(\boldsymbol{\sigma}) .
$$

If $\alpha$ is sufficiently small, the minimum energy configurations of the system are those where $m_{\mu}(\sigma)=1$ for some $\mu$ ('pure states'), which implies that $\sigma=\left(\xi_{1}^{\mu}, \ldots, \xi_{N}^{\mu}\right)$ and pattern $\mu$ is said to be retrieved perfectly. But what does retrieval mean in our immunological context? If $m_{\mu}(\boldsymbol{\sigma})=1$, all the helpers are 'aligned' with their coupled cytokines: those $i$ that inhibit clone $\mu$ (i.e. secrete $\left.\xi_{i}^{\mu}=-1\right)$ will be quiescent $\left(\sigma_{i}=-1\right)$, and those $i$ that excite clone $\mu$ (i.e. secrete $\left.\xi_{i}^{\mu}=1\right)$ will be active $\left(\sigma_{i}=1\right)$ and release the eliciting cytokine. As a result the B-clone $\mu$ receives the strongest possible positive signal (i.e. the random environment becomes a 'staggered magnetic field'), hence it is forced to expand. Thus the arrangement of helper cells leading to the retrieval of pattern $\mu$ corresponds to clone-specific excitatory signalling upon the B-clone $\mu$.

However, if all $\xi_{i}^{\mu} \in\{-1,1\}$ so the bipartite network is fully connected, it can expand only one B-clone at a time. This would be a disaster for the immune system. We need the dilution in the bipartite $\mathrm{B}-\mathrm{H}$ network that is caused by having also $\xi_{i}^{\mu}=0$ (i.e. no signalling between helper $i$ and clone $\mu$ ), to enable multiple clonal expansions. The associative network (3) now involves patterns with blank entries, and 'pure states' no longer work as low energy configurations. Retrieving a pattern no longer employs all spins $\sigma_{i}$, and those corresponding to null entries can be used to recall other patterns. This is energetically favourable since the energy is quadratic in the magnetizations $m_{\mu}(\boldsymbol{\sigma})$. Conceptually, this is only a reshaping of the network's recall tasks: no theoretical bound for information content is violated, and global retrieval is still performed through $N_{B}$ bits. However, the perspective is shifted: the system no longer requires a sharp resolution in information exchange between a helper clone and a B-clone ${ }^{9}$. It suffices that a B-clone receives an attack signal, which could be encoded even by a single bit. In a diluted bipartite B-H system the associative capabilities of the helper network are distributed, in order to simultaneously manage the whole ensemble of B-cells. The analysis of these immunologically most relevant pattern-diluted versions of associative networks is still at an early stage. So far only the low-storage case $N_{B} \sim \log N_{T}$ has been solved $[30,31]$. In this paper we analyse the extreme dilution regime for the B-H system, i.e. $N_{B} \sim N_{T}^{\delta}$ with $0<\delta \leqslant 1$.

\section{Topological properties of the emergent networks}

\subsection{Definitions and simple characteristics}

We start with the definition of the bipartite graph, which contains two sets of nodes (or vertices): the set $V_{B}$ representing B-cells (labelled by $\mu$ ) and the set $V_{T}$ representing T-cells (labelled by $i$ ), of cardinality $N_{B}$ and $N_{T}$, respectively. Nodes belonging to different sets can be pairwise connected via links, which are identically and independently drawn with probability $p$, in such a way that a random bipartite network $\mathcal{B}$ is built. We associate with each link a weight, which can be either +1 or -1 ; these weights are quenched and drawn randomly from a uniform distribution. As a result, the state of each link connecting the $\mu$ th B-clone and the

9 In fact, the high-resolution analysis is performed in the antigenic recognition on the B-cell surface, which is based on a sharp key-and-lock mechanism [2]. 
$i$ th T-clone can be denoted by a random variable $\xi_{i}^{\mu}$, distributed independently according to

$$
P\left(\xi_{i}^{\mu}\right)=\frac{p}{2}\left(\delta_{\xi_{i}^{\mu}, 1}+\delta_{\xi_{i}^{\mu},-1}\right)+(1-p) \delta_{\xi_{i}^{\mu}, 0} .
$$

We choose $p=c / N_{T}^{\gamma}$, with $\gamma \in[0, \infty)$ subject to $p \leqslant 1$, and $c=O\left(N_{T}^{0}\right)$. Upon tuning $\gamma, \mathcal{B}$ displays different topologies, ranging from fully connected (all $N_{T} \times N_{B}$ possible links are present, for $\gamma \rightarrow 0$ ) to fully disconnected (for $\gamma \rightarrow \infty$ ). We have shown in the previous section how a process on this bipartite graph can be mapped to a thermodynamically equivalent process on a new graph, built only of the $N_{T}$ nodes in $V_{T}$, occupied by spins $\sigma_{i}$ that interact pairwise through a coupling matrix with (correlated) entries

$$
J_{i j}=\sum_{\mu=1}^{N_{B}} \xi_{i}^{\mu} \xi_{j}^{\mu} .
$$

The structure of the marginalized system is represented by a weighted monopartite graph $\mathcal{G}$, with weights (6), whose topology is controlled by $\gamma$. To illustrate this, let us consider the weight distribution $P\left(J \mid N_{B}, N_{T}, \gamma, c\right)$, which can be interpreted as the probability distribution for the end-to-end distance of a one-dimensional random walk. This walk has a waiting probability $p_{w}=1-p$, and probabilities of moving left $\left(p_{l}\right)$ or right $\left(p_{r}\right)$ equal to $p_{l}=p_{r}=p / 2$, i.e.

$$
p_{w}=1-\left(c / N_{T}^{\gamma}\right)^{2}, \quad p_{l}=p_{r}=\frac{1}{2}\left(c / N_{T}^{\gamma}\right)^{2} .
$$

Therefore, we can write

$P\left(J \mid N_{B}, N_{T}, \gamma, c\right)=\sum_{S=0}^{L-J} \frac{N_{B} !}{S !\left(\frac{N_{B}-S-J}{2}\right) !\left(\frac{N_{B}-S+J}{2}\right) !} p_{w}^{S} p_{r}^{\left(N_{B}-S+J\right) / 2} p_{l}^{\left(N_{B}-S-J\right) / 2}$,

where the prime indicates that the sum runs only over values of $S$ with the same parity as $N_{B} \pm J$. The result (8) can easily be generalized to the case of biased weight distributions [52], which would correspond to non-isotropic random walks. The first two moments of (8) are, as confirmed numerically in figure 3 :

$$
\langle J\rangle=0, \quad\left\langle J^{2}\right\rangle=\left(c / N_{T}^{\gamma}\right)^{2} N_{B}
$$

We now fix a scaling law for $N_{B}$, namely $N_{B}=\alpha N_{T}^{\delta}$, with $\alpha>0$. This includes the high-load regime for $\delta=1$, as well as the medium-load regime for $\delta \in(0,1)$. The low-storage regime $\delta=0$ has already been treated elsewhere [30,31]. We then find

$$
\left\langle J^{2}\right\rangle=\alpha c^{2} / N_{T}^{2 \gamma-\delta}
$$

The probability of having $J \neq 0$ scales like $N_{T}^{\delta-2 \gamma}$ for $2 \gamma>\delta$, while for $2 \gamma<\delta$ it approaches 1 in the limit $N_{T} \rightarrow \infty$. One can recover the same result via a simple approximation, which is valid in the case $p_{r} \ll 1$ :

$$
P\left(J=0 \mid N_{B}, N_{T}, \gamma, c\right) \approx p_{w}^{N_{B}}=\left[1-\left(c / N_{T}^{\gamma}\right)^{2}\right]^{N_{B}},
$$

see also section A.1 in the appendix for a more rigorous derivation of $P\left(J=0 \mid N_{B}, N_{T}, \gamma, c\right)$. Given the assumed scaling of $N_{B}$, we get $P\left(J \neq 0 \mid N_{B}\left(\alpha, \delta, N_{T}\right), N_{T}, \gamma, c\right) \approx 1-\mathrm{e}^{-\alpha c^{2} N_{T} \delta-2 \gamma}$, which translates into

$$
P\left(J \neq 0 \mid N_{B}\left(\alpha, \delta, N_{T}\right), N_{T}, \gamma, c\right) \approx \begin{cases}\alpha c^{2} N_{T}^{\delta-2 \gamma} & \text { if } 2 \gamma>\delta \\ 1-\mathrm{e}^{-\alpha c^{2}} & \text { if } 2 \gamma=\delta \\ 1 & \text { if } 2 \gamma<\delta .\end{cases}
$$



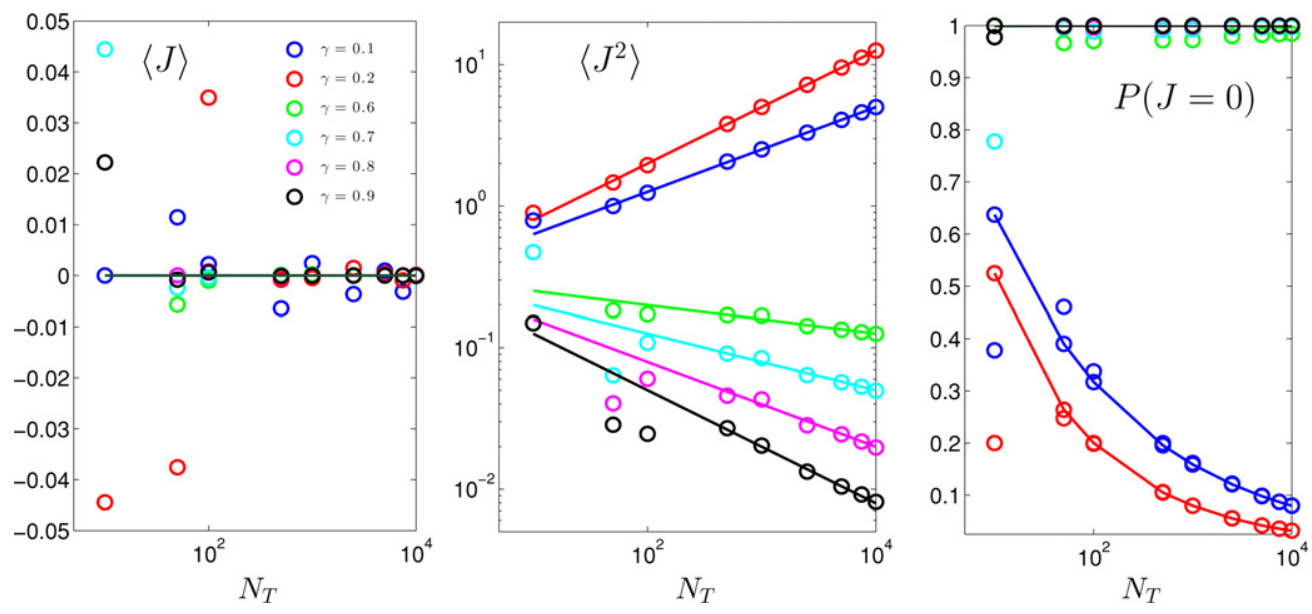

Figure 3. Statistical properties of individual links in randomly generated instances of the graph $\mathcal{G}$ at different sizes $N_{T}$, with $N_{B}=\alpha N_{T}^{\delta}$. We measured the mean coupling $\langle J\rangle$ (left), the mean squared coupling $\left\langle J^{2}\right\rangle$ (middle) and the probability $P(J=0)$ of a zero link (right), for different values of $\gamma$. The parameters $\delta=1$ and $\alpha=0.5$ are kept fixed. Solid lines: predictions given in (9) and (12). Markers: simulation data.

This quantity can be interpreted as the average link probability in $\mathcal{G}$. The average degree $\bar{z}$ over the whole set of nodes ${ }^{10}$ can then be written as

$$
\bar{z}=N_{T} P(J \neq 0) \text {. }
$$

Thus, if we adopt a mean-field approach based only on the estimates (12), (13), we find that $\mathcal{G}$ can display the following topologies, expressed in terms of the average degree $\bar{z}$ of $\mathcal{G}$ (the average number of links per node).

\begin{tabular}{lll}
\hline & $0<\gamma<1$ & $\gamma=1$ \\
\hline$\delta<2 \gamma-1$ & Fully disconnected, $\bar{z} \rightarrow 0$ & Fully disconnected, $\bar{z} \rightarrow 0$ \\
$\delta=2 \gamma-1$ & Finitely connected, $\bar{z}=O(1)$ & Finitely connected, $\bar{z}=O(1)$ \\
$2 \gamma-1<\delta<2 \gamma$ & Extremely diluted, $\bar{z} \rightarrow \infty$ but $\bar{z} / N_{T} \rightarrow 0$ & - \\
$\delta=2 \gamma$ & Finitely diluted, $\bar{z}=O\left(N_{T}\right)$ & - \\
$\delta>2 \gamma$ & Fully connected, $\bar{z}=N_{T}$ & - \\
\hline
\end{tabular}

The missing entries in the table correspond to forbidden values $\delta \notin(0,1]$. The various cases are also summarized in the left panel of figure 4 . This picture is confirmed by numerical simulations. The right panels of figure 4 give a finite-size scaling analysis for the average degree $\bar{z}$ and its fluctuations $\bar{z}^{2}-\bar{z}^{2}$, measured in realizations of $\mathcal{G}$ for several choices of parameters. We also show corresponding data for Erdös-Rényi graphs, where all links are independently drawn with probability $q$, for comparison (here $\bar{z}=q N_{T}$ and $\bar{z}^{2}-\bar{z}^{2}=N_{T} q(1-q)$ ). We find that (i) in the fully disconnected regime of the phase diagram, $\bar{z}$ decays to zero exponentially as a function of $N_{T}$, (ii) in the extremely diluted regime, $\bar{z}$ scales with $N_{T}$ according to a power law, (iii) in the finitely diluted regime $\bar{z}$ is proportional to $N_{T}$, and (iv) in the fully connected

${ }^{10}$ The degree or coordination number $z_{i}$ of node $i$ is the number of its nearest-neighbours, i.e. the number of links stemming from the node itself. Thus, the average degree $\bar{z}=\sum_{i \in V_{T}} z_{i} / N_{T}$ measures the density of links present in the graph. 

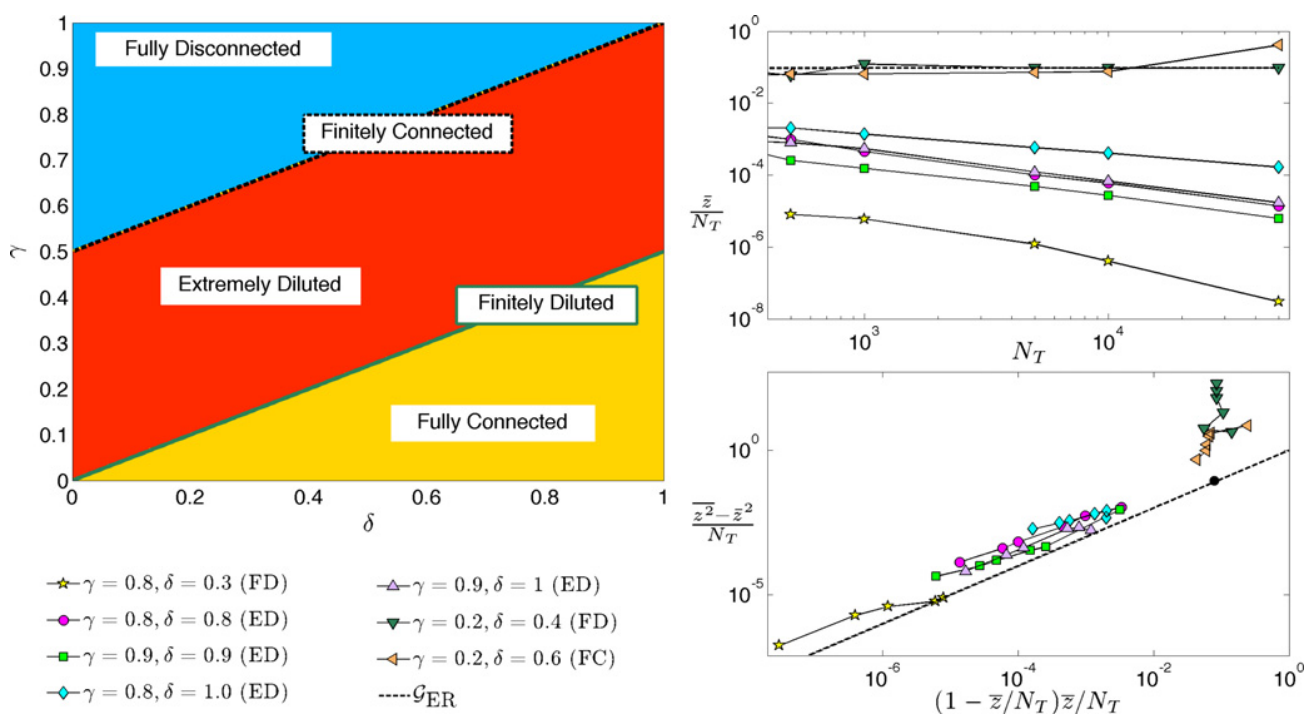

Figure 4. Left: qualitative phase diagram describing the different topological regimes of $\mathcal{G}$, according to the mean-field analysis in section 3. Right panels: finite-size scaling for the average degree $\bar{z}$ (upper) and fluctuations $\bar{z}^{2}-\bar{z}^{2}$ (lower), measured on realizations of $\mathcal{G}$ for different choices of parameters $\gamma$ and $\delta$ (see legend). The parameters $c=1$ and $\alpha=0.1$ are kept fixed. The markers correspond to numerical data, and the lines connecting the markers are guides to the eye. The cases being compared include fully disconnected (FD), extremely diluted (ED), finitely diluted (FD) and fully connected (FC) regimes, and they are shown together with data on Erdös-Rènyi graphs $\mathcal{G}_{\text {ER }}$ with link probability $q=1-\mathrm{e}^{\alpha c^{2}}$ (dashed lines, see equation (12)). For ER graphs one expects $\bar{z} / N_{T}$ to be constant, and equal to $q$, while the normalized fluctuations are expected to be $\left(1-\bar{z} / N_{T}\right) \bar{z} / N_{T}$. Different points pertain to different graph sizes, but with the same link probability $q$; they are found to overlap regardless of their size $(\bullet)$. For $\mathcal{G}$, the behaviour of $\bar{z}$ is consistent with mean-field expectations, while connectivity fluctuations are underestimated by the mean-field approach.

regime $\bar{z}$ saturates to $N_{T}$. There is thus full agreement with the predictions of the mean-field approach. The fluctuations are slightly larger that those of a purely randomly drawn network, which suggests that $\mathcal{G}$ exhibits a certain degree of inhomogeneity. This will be investigated next.

It is important to stress that, as the system parameters $\gamma$ and $\delta$ are tuned, the connectivity of the resulting network $\mathcal{G}$ can vary extensively and therefore, in order for the Hamiltonian (4) to scale linearly with the system size $N_{T}$, the prefactor $1 / N_{T}$ of the Hopfield model embedded in complete graphs, is not generally appropriate. One should normalize $\mathcal{H}(\boldsymbol{\sigma} \mid \xi)$ according to the expected connectivity of the graph.

\subsection{Component size distribution}

As $\gamma$ is increased, both $\mathcal{B}$ and $\mathcal{G}$ become more and more diluted, eventually under-percolating. The topology analysis can be carried out more rigorously for the (bipartite) graph $\mathcal{B}$, since its link probability $p=c / N_{T}^{\gamma}$ is constant and identical for all $(i, \mu)$. We can apply the generating function formalism developed in $[53,54]$ to show that the size of the giant component $\left(\subseteq V_{T}, V_{B}\right)$ diverges when

$$
p^{2}=1 / N_{B} N_{T}
$$



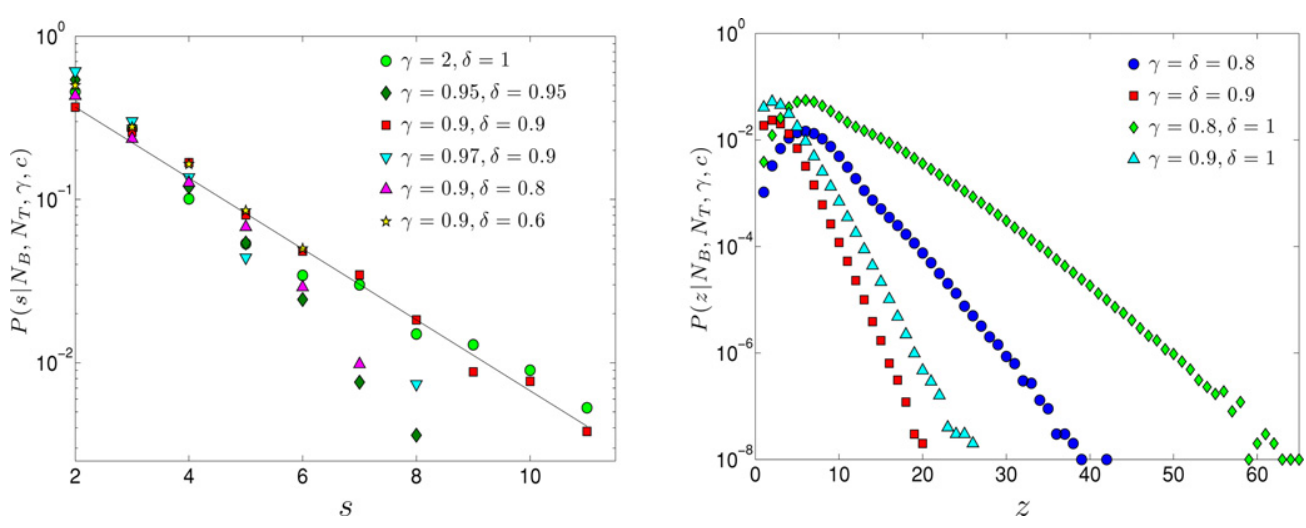

Figure 5. Left: distribution of component sizes of the graph $\mathcal{G}$, for $\delta=c=1, \alpha=0.1$ and different values of $\gamma$. Symbols represent simulation data; the solid line represents the analytical estimate for the underpercolated case ( $\square$ ). Right: degree distributions obtained for different values of $\delta$ and $\gamma$, with $\alpha=0.1$ and $c=1 \mathrm{kept}$ fixed. The semi-logarithmic scale highlights the exponential decay at large values of $z$.

Hence, upon setting $N_{B}=\alpha N_{T}^{\delta}$, the percolation threshold for the bipartite graph $\mathcal{B}$ is defined by

$$
N_{T}^{2 \gamma-1-\delta}=c^{2} \alpha=O\left(N_{T}^{0}\right) \Rightarrow \gamma=(\delta+1) / 2,
$$

which is consistent with the results of section 3; we refer to section A.2 in the appendix for full details. Below the percolation threshold the generating function formalism also allows us to get the distribution $P_{\mathcal{B}}\left(s \mid N_{T}, N_{B}, c, \gamma\right)$ for the size $s$ of the small components occurring in $\mathcal{B}$. A (connected) component of an undirected graph is an isolated subgraph in which any two vertices are connected to each other by paths; the size of the component is simply the number of nodes belonging to the component itself. We prove in section A.2 in the appendix that just below the percolation threshold, $P_{\mathcal{B}}\left(s \mid N_{B}, N_{T}, c, \gamma\right)$ scales exponentially with $s$. One finds that this is true also for the distribution $P_{\mathcal{G}}\left(s \mid N_{B}, N_{T}, c, \gamma\right)$ of graph $\mathcal{G}$ (see figure 5, left panel).

Interestingly, the small components of $\mathcal{G}$ that emerge around and below the percolation threshold play a central role in the network's retrieval performance. To see this, one may consider the extreme case where the bipartite graph $\mathcal{B}$ consists of trimers only. Here each node $\mu \in V_{B}$ is connected to two nodes $i_{1}, i_{2} \in V_{T}$, that is $\left|\xi_{i_{1}}^{\mu}\right|=\left|\xi_{i_{2}}^{\mu}\right|=1$ and $\xi_{i_{1}}^{\nu}=\xi_{i_{2}}^{\nu}=0, \forall \nu \neq \mu$. The associated graph $\mathcal{G}$ is then made up of dimers $\left(i_{1}, i_{2}\right)$ only, and $J_{i j} \in\{-1,0,1\}$ for all $(i, j)$. The energetically favourable helper cell configuration $\sigma$ is now the one where $\sum_{i} \xi_{i}^{\mu} \sigma_{i}= \pm 2$, for any $\mu$. This implies that retrieval of all patterns is accomplished (under proper normalization). In the opposite extreme case, $\mathcal{B}$ is fully connected, and the helper cell system becomes a Hopfield network where parallel retrieval is not realized. In general, around and below the percolation threshold, the matrix $\xi$ turns out to be partitioned, which implies that also the coupling matrix $\mathbf{J}$ is partitioned, and each block of $\mathbf{J}$ corresponds to a separate component of the overall graph $\mathcal{G}$. For instance, looking at the bipartite graph $\mathcal{B}$, a star-like module with node $\mu \in V_{B}$ at its centre and the nodes $i_{1}, i_{2}, \ldots, i_{n} \in V_{T}$ as leaves ${ }^{11}$ can occur when the leaves share a unique non-null $\mu$ th entry in their patterns, that is $\left|\xi_{i_{1}}^{\mu}\right|=\left|\xi_{i_{2}}^{\mu}\right|=\cdots=\left|\xi_{i_{n}}^{\mu}\right|=1$. For the graph $\mathcal{G}$ this module corresponds to a complete sub-graph $K_{n}$ of $n \leqslant N_{T}$ nodes. In this case the retrieval of pattern $\mu$ is trivially achieved. In fact, a complete sub-graph $K_{n}$ in $\mathcal{G}$ can originate from more general arrangements in $\mathcal{B}$ :

${ }^{11}$ The opposite case of a star-like module with the centre belonging to $V_{T}$ is unlikely, given that $N_{T}>N_{B}$. 
each leaf $i$ can display several other null entries beyond $\mu$, but these are not shared, that is $\xi_{i}^{v} \xi_{j}^{v}=0 \forall j \in V_{T}, v \neq \mu \in V_{B}$. For instance, all stars with centres belonging to $V_{B}$ and with leaves of length 1 or 2 fall into this extended class. Again, the retrieval of pattern $\mu$ and possibly of further patterns $v$ is achieved. However, the mutual signs of magnetizations are no longer arbitrary, as the terms $m_{\mu} \xi_{i}^{\mu}=m_{\nu} \xi_{i}^{\nu}$ are subject to constraints.

We can further generalize the topology of components in $\mathcal{G}$ compatible with parallel retrieval, by considering cliques (i.e. subsets of nodes such that every two nodes in the subset are connected by an edge), which are joined together by one link: each clique consists of nodes $\in V_{T}$ that share the same non-null entry, so that the unique link between two cliques is due to a node displaying at least two non-null entries. This kind of structure exhibits a high degree of modularity; each clique is a module and corresponds to a different pattern. As for retrieval, this arrangement works fine as there is no interference between the signal on each node in $V_{T}$. For this arrangement to occur a sufficient condition is that $\mathcal{B}$ is devoid of squares, so that two nodes $\in V_{T}$ do not share more than one neighbour. This implies that, among the $n$ nodes connected to $j \in V_{B}$, the probability that any number $k>2$ of these display another common neighbour is vanishing:

$$
\lim _{N_{T} \rightarrow \infty} \sum_{k>2}^{n}\left(\begin{array}{c}
n \\
k
\end{array}\right) p^{k}(1-p)^{n-k}=\lim _{N_{T} \rightarrow \infty}\left\{1-\left(1-\frac{c}{N^{\gamma}}\right)^{n}-\frac{c n}{N^{\gamma}}\left(1-\frac{c}{N^{\gamma}}\right)^{n-1}\right\}=0 .
$$

Since $n \sim N_{T}^{\delta-\gamma}$, we obtain the condition $\gamma \geqslant \delta$. Examples of numerically generated graphs $\mathcal{G}$, for different choices of parameters, are shown in figure 6 .

\subsection{Clustering properties}

We saw that in the operationally most important parameter regime the graph $\mathcal{G}$ is built of small cliques which are poorly (if at all) connected to each other. This means that non-isolated nodes are highly clustered and the graph will have a high degree of modularity, i.e. dense connections between nodes within the same 'module', but sparse connections between nodes in different 'modules'. The clustering coefficient $C_{i}$ of a node $i$ measures how close its $z_{i}$ neighbours are to being a clique. It is defined as

$$
C_{i}=2 E_{i} / z_{i}\left(z_{i}-1\right),
$$

where $E_{i}$ is the number of links directly connecting nodes pairs in $V_{i}$, while $\frac{1}{2} z_{i}\left(z_{i}-1\right)$ is the total number of non-ordered node pairs in $V_{i}$. Hence $C_{i} \in[0,1]$. The average clustering coefficient $\bar{C}=N_{B, T}^{-1} \sum_{i \in V_{B, T}} C_{i}$ measures the extent to which nodes in a graph tend to cluster together. It is easy to see that for a bipartite graph, by construction, $C_{i}=0$ for any node, while for homogeneous graphs the local clustering coefficients are narrowly distributed around $\bar{C}$. For instance, for the Erdös-Rény random graph, where links are identically and independently drawn with probability $q$, the local coefficients are peaked around $q$. As for our graphs $\mathcal{G}$, due to their intrinsic inhomogeneity, the global measure $\bar{C}$ would give only limited information. In contrast, the distribution $P\left(C \mid N_{B}, N_{T}, \gamma, c\right)$ of local clustering coefficients informs us about the existence and extent of cliques or 'bulk' nodes, which would be markers of low and high recall interference, respectively. Indeed, as shown in figure 7, in the highly diluted regime most of the nodes in $\mathcal{G}$ are either highly clustered, i.e. exhibiting $C_{i}=1$, or isolated, with $C_{i}=0$, whereas the coefficients of the remaining nodes are distributed around intermediate values with average decreasing with $\gamma$, as expected. In particular, when both $\delta$ and $\gamma$ are relatively large, $P\left(C \mid N_{B}, N_{T}, \gamma, c\right)$ approaches a bimodal distribution with peaks at $C=0$ and $C=1$, whereas when $\delta$ is sufficiently larger than $\gamma$, there exists a fraction of nodes with intermediate clustering which make up a bulk. Therefore, although the density of links is rather small, the 

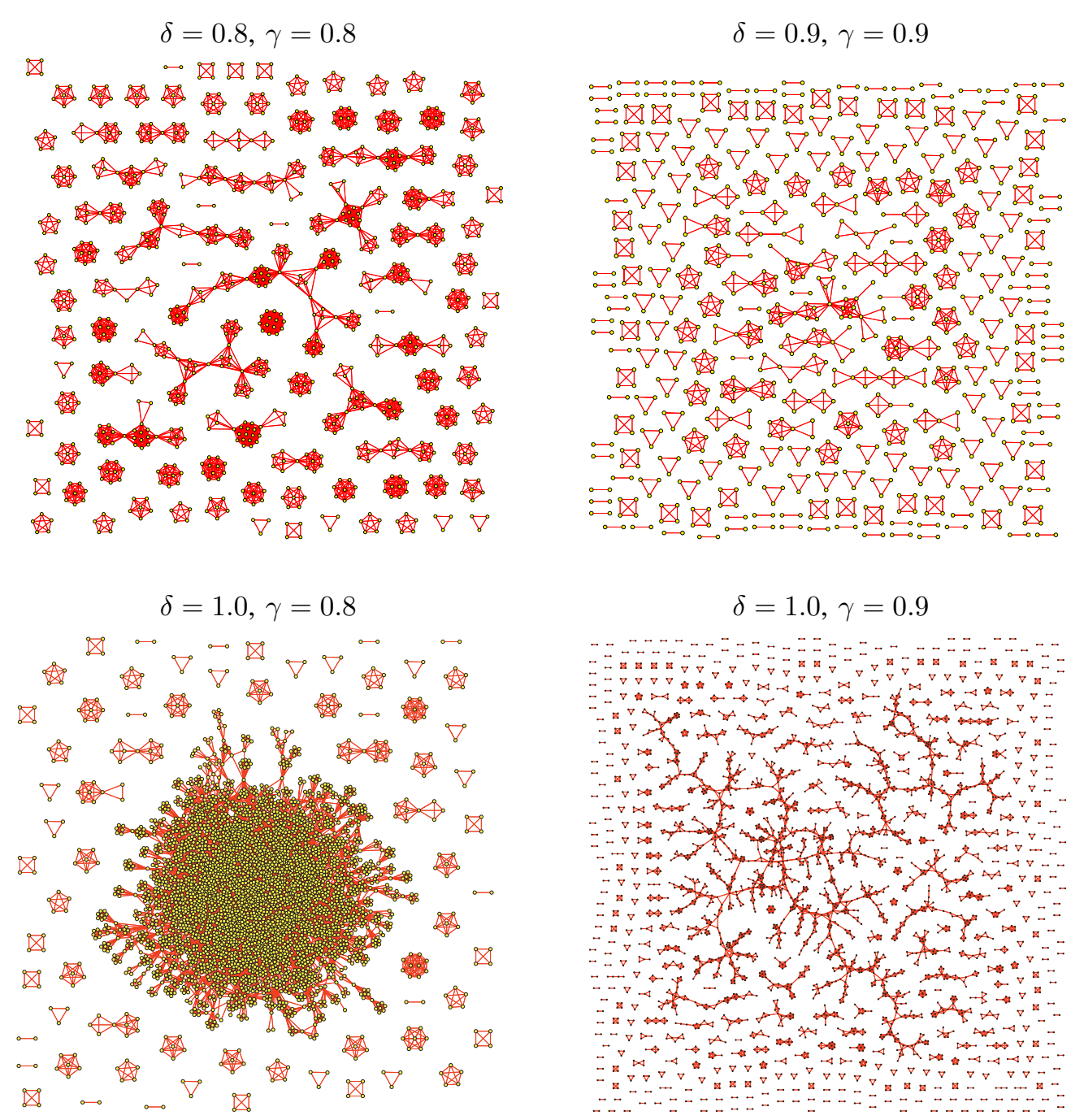

Figure 6. Plots of numerically generated graphs $\mathcal{G}$ for $N_{T}=10^{4}$ and $\alpha=0.1$, and different combinations of $(\delta, \gamma)$. All isolated nodes have been omitted from the plots. The chosen parameter combinations all give graphs that are just below the percolation threshold $\delta=2 \gamma-1$. However, the two graphs at the top satisfy the further condition $\delta \leqslant \gamma$ that marks the ability of simultaneous pattern retrieval (via weakly connected small cliques). In the bottom graphs this latter condition is violated, so these would behave more like conventional Hopfield networks (here simultaneous retrieval of multiple patterns is not possible).

average clustering coefficient is very high, and this is due to the fragmentation of the graph into many small cliques.

To measure the extent of modular structures we constructed the topological overlap matrix $\mathbf{T}$, whose entry $T_{i j}=\sum_{k \neq i, j} c_{i k} c_{j k} / z_{i} \in[0,1]$ returns the normalized number of neighbours that $i$ and $j$ share. The related patterns for several choices of parameters are shown in the plots of figure 8, and compared to those of Erdös-Rènyi graphs $\mathcal{G}_{\text {ER }}$. For Erdös-Rènyi graphs $\mathbf{T}$ displays a homogeneous pattern, that is very different from the highly clustered cases emerging from $\mathcal{G}$. In particular, for the highly diluted cases considered here, we find that smaller values of $\gamma$ induce a smaller number of modules, that are individually increasing in size. 

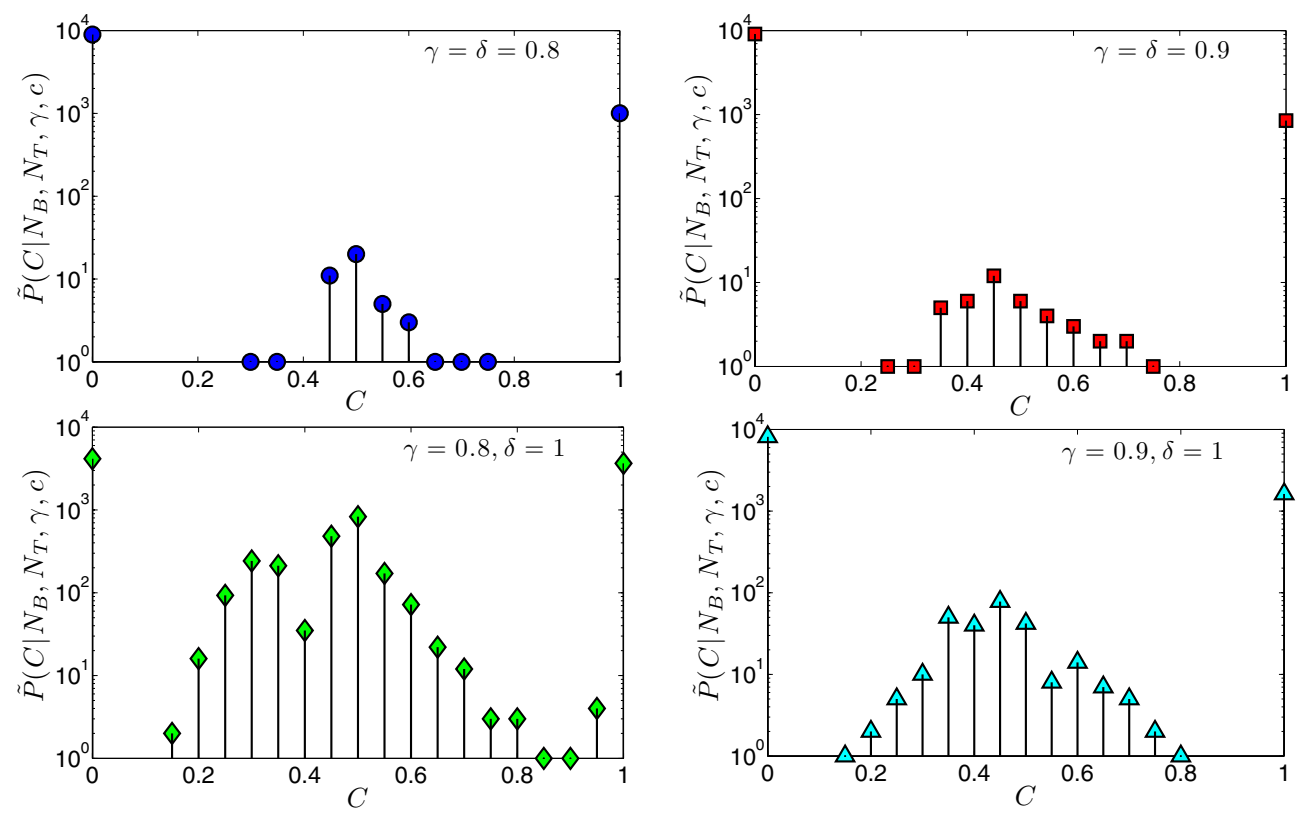

Figure 7. Histograms for $\tilde{P}\left(C \mid N_{B}, N_{T}, \gamma, c\right)$, normalized such that $\tilde{P}\left(C \mid N_{B}, N_{T}, \gamma, c\right)=1$ for those values of $C$ that have the lowest occurrence frequency. This normalization allows to highlight the intermediate region, where the frequencies are much lower than those pertaining to $C=0$ and $C=1$. The results shown here refer to graphs with $N_{T}=10^{4}$ nodes, $\alpha=0.1$ and $c=1$, while $\delta$ and $\gamma$ are varied.

\section{Medium storage regime in extremely diluted connectivity: retrieval region}

We now turn to the statistical mechanics analysis, and consider the immune network model composed of $N_{T}$ T-clones $\left(\sigma_{i}, i=1, \ldots, N_{T}\right)$ and $N_{B}$ B-clones $\left(b_{\mu}, \mu=1, \ldots, N_{B}\right)$, such that the number ratio scales as

$$
\lim _{N_{T} \rightarrow \infty} N_{B} / N_{T}^{\delta}=\alpha, \quad \delta \in(0,1), \quad \alpha>0 .
$$

The effective interactions in the reduced network with helper cells only are described by the Hamiltonian

$$
\mathcal{H}(\boldsymbol{\sigma} \mid \xi)=-\frac{1}{2 N_{T}^{\tau}} \sum_{i, j=1}^{N_{T}} \sum_{\mu=1}^{N_{B}} \xi_{i}^{\mu} \xi_{j}^{\mu} \sigma_{i} \sigma_{j},
$$

where the cytokine components $\xi_{i}^{\mu} \in\{0, \pm 1\}$ are quenched random variables, independently and identically distributed according to

$$
P\left(\xi_{i}^{\mu}=1\right)=P\left(\xi_{i}^{\mu}=-1\right)=c / 2 N_{T}^{\gamma}, \quad P\left(\xi_{i}^{\mu}=0\right)=1-c / N_{T}^{\gamma}
$$

with $\gamma \in[0,1)$. The parameter $\tau$ must be chosen such that $\mathcal{H}(\sigma \mid \xi)$ scales linearly with $N_{T}$, and must therefore depend on $\gamma$ and $\delta$. Heuristically, since the number of non-zero entries $\mathcal{N}_{\text {nz }}$ in a generic pattern $\left(\xi_{1}^{\mu}, \ldots, \xi_{N_{T}}^{\mu}\right)$ is $O\left(N_{T}^{1-\gamma}\right)$, we expect that the network can retrieve a number of patterns of order $O\left(N_{T} / \mathcal{N}_{\mathrm{nz}}\right)=O\left(N_{T}^{\gamma}\right)$. We therefore expect to see changes in $\tau$ only when crossing the region in the $(\gamma, \delta)$ plane where pattern sparseness prevails over storage load (i.e. $\delta<\gamma$, where the system can recall all patterns), to the opposite situation, 

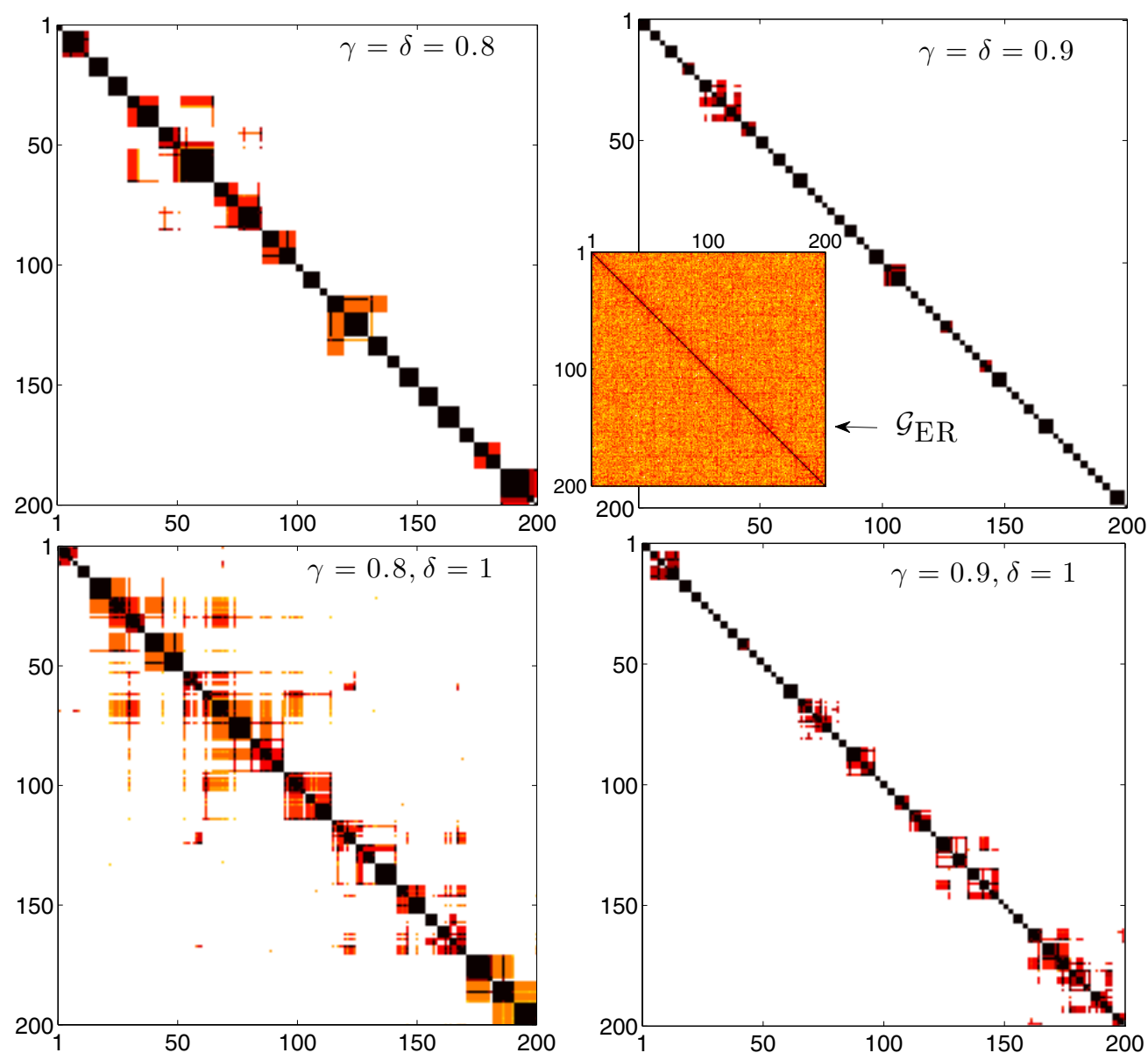

Figure 8. Overlap matrix $\mathbf{T}$ with entries $T_{i j}=\sum_{k \neq i, j} c_{i k} c_{j k} / z_{i}$. The nodes are ordered such that nodes with large overlaps are adjacent, and the most significant part of $\mathbf{T}$ is around the diagonal. Note: $T_{i i}=1$ for all $i$, by construction. Darker colours correspond to larger entries, and any extended coloured zone denotes a module, i.e. a set of nodes that are highly clustered and possibly not connected with the remaining nodes. These plots refer to numerically generated graphs with $N_{T}=10^{4}$ nodes, $\alpha=0.1$ and $c=1$, while $\delta$ and $\gamma$ are varied. To avoid cluttering of the figures, only a fraction $200 \times 200$ of each pattern is shown. A similar fraction of the overlap matrix for an extremely diluted Erdös-Rènyi graph made of $10^{4}$ nodes is also depicted for comparison (inset). In this case there is no evidence of modularity; $\mathbf{T}$ displays a homogeneous pattern.

where the load is too high and frustration by multiple inputs on the same entry drives the network to saturation (i.e. $\delta>\gamma$ ). To validate this scenario, which is consistent with our previous topological investigation, we carry out a statistical mechanical analysis, based on computing the free energy

$$
f(\beta)=-\lim _{N_{T} \rightarrow \infty} \frac{1}{\beta N_{T}}\left\langle\log Z_{N_{T}}(\beta, \xi)\right\rangle_{\xi}
$$




\subsection{Free energy computation and physical meaning of the parameters}

If the number of patterns is sufficiently small compared to $N_{T}$, i.e. $\delta<1$, we do not need the replica method; we can simply apply the steepest descent technique using the $N_{B} \ll N_{T} / \log N_{T}$ Mattis magnetizations as order parameters:

$f(\beta)=-\frac{1}{\beta} \log 2-\lim _{N_{T} \rightarrow \infty} \frac{1}{\beta N_{T}} \log \int \mathrm{d} \boldsymbol{m} \exp \left(-\frac{1}{2} \boldsymbol{m}^{2}+N_{T}\left\langle\log \cosh \left(\sqrt{\beta / N_{T}^{\tau}} \boldsymbol{\xi} \cdot \boldsymbol{m}\right)\right\rangle_{\xi}\right)$

with $\boldsymbol{m}=\left(m_{1}, \ldots, m_{N_{B}}\right), \boldsymbol{\xi}=\left(\xi^{1}, \ldots, \xi^{N_{B}}\right)$ and $\boldsymbol{\xi} \cdot \boldsymbol{m}=\sum_{\mu} \xi^{\mu} m_{\mu}$. Rescaling of the order parameters via $m_{\mu} \rightarrow m_{\mu} \sqrt{\beta} c N_{T}^{\tau / 2+\theta}$ then gives

$$
\begin{aligned}
f(\beta)=-\frac{1}{\beta} \log 2 & -\lim _{N_{T} \rightarrow \infty} \frac{1}{\beta N_{T}} \log \int \mathrm{d} \boldsymbol{m} \\
& \times \exp \left(N_{T}\left(-\frac{\beta c^{2}}{2} N_{T}^{\tau+2 \theta-1} \boldsymbol{m}^{2}+\left\langle\log \cosh \left(\beta c N_{T}^{\theta} \boldsymbol{\xi} \cdot \boldsymbol{m}\right)\right\rangle_{\xi}\right)\right) .
\end{aligned}
$$

Hence, provided the limit exists, we may write via steepest descent integration:

$f(\beta)=-\frac{1}{\beta} \log 2-\frac{1}{\beta} \lim _{N_{T} \rightarrow \infty} \operatorname{extr}_{\boldsymbol{m}}\left[\left\langle\log \cosh \left(\beta c N_{T}^{\theta} \boldsymbol{\xi} \cdot \boldsymbol{m}\right)\right\rangle_{\xi}-\frac{\beta c^{2}}{2} N_{T}^{\tau+2 \theta-1} \boldsymbol{m}^{2}\right]$.

Differentiation with respect to the $m_{\mu}$ gives the self-consistent equations for the extremum:

$$
m_{\mu}=\frac{N_{T}^{1-\tau-\theta}}{c}\left\langle\xi^{\mu} \tanh \left(\beta c N_{T}^{\theta} \boldsymbol{\xi} \cdot \boldsymbol{m}\right)\right\rangle_{\xi} .
$$

With the additional new parameter $\theta$, we now have two parameters with which to control separately two types of normalization: the normalization of the Hamiltonian, via $\tau$, and the normalization of the order parameters, controlled by $\theta$. To carry out this task properly, we need to understand the physical meaning of the order parameters. This is done in the usual way, by adding suitable external fields to the Hamiltonian:

$$
\mathcal{H} \rightarrow \mathcal{H}-\sum_{\mu=1}^{N_{B}} \lambda_{\mu} \sum_{i=1}^{N_{T}} \xi_{i}^{\mu} \sigma_{i}
$$

Now, with $\langle g(\sigma)\rangle_{\sigma}=Z_{N_{T}}^{-1}(\beta, \xi) \sum_{\sigma} \mathrm{e}^{-\beta \mathcal{H}(\sigma \mid \xi)} g(\boldsymbol{\sigma})$ and the corresponding new free energy $f(\beta, \lambda)$,

$$
\lim _{N_{T} \rightarrow \infty} \frac{1}{N_{T}}\left\langle\sum_{i=1}^{N_{T}} \xi_{i}^{\mu} \sigma_{i}\right\rangle_{\sigma}=-\left.\frac{\partial f(\beta, \lambda)}{\partial \lambda_{\mu}}\right|_{\lambda=0},
$$

with the short-hand $\lambda=\left(\lambda_{1} \ldots, \lambda_{N_{B}}\right)$. The new free energy is then found to be

$f(\beta, \lambda)=-\frac{1}{\beta} \log 2-\frac{1}{\beta} \lim _{N_{T} \rightarrow \infty} \operatorname{extr}_{\boldsymbol{m}}\left[\left\langle\log \cosh \left(\beta \xi \cdot\left[c N_{T}^{\theta} \boldsymbol{m}+\lambda\right]\right)\right\rangle_{\xi}-\frac{\beta c^{2}}{2} N_{T}^{\tau+2 \theta-1} \boldsymbol{m}^{2}\right]$.

Upon differentiation with respect to $\lambda_{\mu}$ we find (27) taking the form

$$
\lim _{N_{T} \rightarrow \infty} \frac{1}{N_{T}}\left\langle\sum_{i=1}^{N_{T}} \xi_{i}^{\mu} \sigma_{i}\right\rangle_{\sigma}=\lim _{N_{T} \rightarrow \infty}\left\langle\xi^{\mu} \tanh \left(\beta c N_{T}^{\theta} \boldsymbol{\xi} \cdot \boldsymbol{m}\right)\right\rangle_{\xi}
$$


We can then use expression (25) for $m_{\mu}$ to obtain the physical meaning of our order parameters:

$$
\begin{aligned}
m^{\mu} & =\lim _{N_{T} \rightarrow \infty} \frac{N_{T}^{1-(\tau+\theta)}}{c}\left\langle\xi^{\mu} \tanh \left(\beta c N_{T}^{\theta} \boldsymbol{\xi} \cdot \boldsymbol{m}\right)\right\rangle_{\xi} \\
& =\lim _{N_{T} \rightarrow \infty}\left\langle\frac{1}{c N_{T}^{\tau+\theta}} \sum_{i=1}^{N_{T}} \xi_{i}^{\mu} \sigma_{i}\right\rangle_{\sigma} .
\end{aligned}
$$

Let us summarize the status of the various remaining control parameters in the theory, in the interest of transparency. Our model has three given external parameters:

- $\gamma \in[0,1)$ : this quantifies the dilution of stored patterns, via $P\left(\xi_{i}^{\mu} \neq 0\right)=c N_{T}^{-\gamma}$,

- $\delta \in(0,1)$ and $\alpha>0$ : these determine the number of stored patterns, via $\lim _{N_{T} \rightarrow \infty} N_{B} / N_{T}^{\delta}=\alpha$.

It also has two 'internal' parameters, which must be set in such a way for the statistical mechanical calculation to be self-consistent, i.e. such that various quantities scale in the physically correct way for $N_{T} \rightarrow \infty$ :

- $\tau \geqslant 0$ : this must ensure that the energy $\mathcal{H}=-\left\langle\frac{1}{2} N_{T}^{-\tau} \sum_{\mu=1}^{N_{B}}\left(\sum_{i=1}^{N_{T}} \xi_{i}^{\mu} \sigma_{i}\right)^{2}\right\rangle_{\sigma}$ scales as $O\left(N_{T}\right)$

- $\theta \geqslant 0$ : this must ensure that the order parameter $m_{\mu}=\left\langle\left(1 / c N_{T}^{\tau+\theta}\right) \sum_{i} \xi_{i}^{\mu} \sigma_{i}\right\rangle_{\sigma}$ are of order $O(1)$.

\subsection{Setting of internal scaling parameters}

To find the appropriate values for the internal scaling parameters $\theta$ and $\tau$ we return to the order parameter equation (25) and carry out the average over $\xi^{\mu}$. This gives

$$
\begin{aligned}
m_{\mu} & =\frac{N_{T}^{1-\tau-\theta}}{c}\left\langle\tanh \left(\beta c N_{T}^{\theta}\left(\left(\xi^{\mu}\right)^{2} m_{\mu}+\xi^{\mu} \sum_{\nu \neq \mu}^{N_{B}} \xi^{\nu} m_{\nu}\right)\right)\right\rangle_{\xi}, \\
& =N_{T}^{1-\tau-\theta-\gamma}\left\langle\tanh \left(\beta c N_{T}^{\theta}\left(m_{\mu}+\sum_{\nu \neq \mu}^{N_{B}} \xi^{\nu} m_{\nu}\right)\right)\right\rangle_{\xi} .
\end{aligned}
$$

Having non-vanishing $m_{\mu}$ in the limit $N_{T} \rightarrow \infty$ clearly demands $\theta+\tau \leqslant 1-\gamma$. If $\theta>0$ the $m_{\mu}$ will become independent of $\beta$, which means that any phase transitions occur ar zero or infinite noise levels, i.e. we would not have defined the scaling of our Hamiltonian correctly. Similarly, if $\theta+\tau<1-\gamma$ the effective local fields acting upon the $\sigma_{i}$ (viz. the arguments of the hyperbolic tangent) and therefore also the expectation values $\left\langle\sigma_{i}\right\rangle_{\sigma}$, would be vanishingly weak. We therefore conclude that a natural ansatz for the free exponents is:

$$
(\tau, \theta)=(1-\gamma, 0)
$$

This simplifies the order parameter equation to

$$
m_{\mu}=\left\langle\tanh \left(\beta c\left(m_{\mu}+\sum_{\nu \neq \mu}^{N_{B}} \xi^{v} m_{\nu}\right)\right)\right\rangle_{\xi} .
$$

Let us analyse this equation further. Since $P\left(\xi_{i}^{\mu} \neq 0\right) \sim N_{T}^{-\gamma}$ with $\gamma>0$, we can for $N_{T} \rightarrow \infty$ replace in (31) the sum over $v \neq \mu$ with the sum over all $\mu$; the difference is negligible in the thermodynamic limit. In this way it becomes clear that for each solution of (31) we have $m_{\mu} \in\{-m, 0, m\}$. Using the invariance of the free energy under $m_{\mu} \rightarrow-m_{\mu}$, we can from 
now on focus on solutions with non-negative magnetizations. If we denote with $K \leqslant N_{B}$ the number of $\mu$ with $m_{\mu} \neq 0$, then the value of $m>0$ is to be solved from

$$
m=\left\langle\tanh \left(\beta c m\left(1+\sum_{\nu=1}^{K} \xi^{\nu}\right)\right)\right\rangle_{\xi} .
$$

It is not a priori obvious how the number $K$ of non-zero magnetizations (i.e. the number of simultaneously triggered clones) can or will scale with $N_{T}$. We therefore set $K=\phi N_{T}^{\delta^{\prime}}$, in which the condition $K \leqslant N_{B}$ then places the following conditions on $\phi$ and $\delta^{\prime}: \delta^{\prime} \in[0, \delta]$, and $\phi \in[0, \infty)$ if $\delta^{\prime}<\delta$ or $\phi \in[0, \alpha]$ if $\delta^{\prime}=\delta$. We expect that if $K$ is too large, equation (35) will only have the trivial solution for $N_{T} \rightarrow \infty$, so there will be further conditions on $\phi$ and $\delta^{\prime}$ for the system to operate properly. If $\delta^{\prime}>\gamma$, the noise due to other condensed patterns (i.e. the sum over $v$ ) becomes too high, and $m$ can only be zero:

$$
\mathbb{E}\left[\left(\sum_{\mu=1}^{K} \xi^{\mu}\right)^{2}\right]=\sum_{\mu=1}^{K} \mathbb{E}\left[\xi^{\mu 2}\right]=\phi c \frac{N_{T}^{\delta^{\prime}}}{N_{T}^{\gamma}} \rightarrow \infty .
$$

On the other hand, if $\delta^{\prime}<\gamma$ this noise becomes negligible, and (35) reduces to the CurieWeiss equation, whose solution is just the Mattis magnetization [35, 51, 55]. It follows that the critical case is the one where when $\delta^{\prime}=\gamma$. Here we have for $N_{T} \rightarrow \infty$ the following equation for $m$ :

$$
m=\sum_{k \in \mathbb{Z}} \pi(k \mid \phi) \tanh (\beta c m(1+k))
$$

with the following discrete noise distribution, which obeys $\pi(-k \mid \phi)=\pi(k \mid \phi)$ :

$$
\pi(k \mid \phi)=\left\langle\delta_{k, \sum_{\mu=1}^{\infty} \xi^{\mu}}\right\rangle_{\xi} .
$$

\subsection{Computation of the noise distribution $\pi(k)$}

Given its symmetry, we only need to calculate $\pi(k \mid \phi)$ for $k \geqslant 0$ :

$$
\begin{aligned}
\pi(k \mid \phi) & =\lim _{K \rightarrow \infty} \int_{-\pi}^{\pi} \frac{\mathrm{d} \psi}{2 \pi} \mathrm{e}^{-\mathrm{i} \psi k}\left\langle\mathrm{e}^{\mathrm{i} \psi \xi}\right\rangle_{\xi}^{K}=\lim _{K \rightarrow \infty} \int_{-\pi}^{\pi} \frac{\mathrm{d} \psi}{2 \pi} \mathrm{e}^{-\mathrm{i} \psi k}\left(1+\frac{c \phi}{K}(\cos \psi-1)\right)^{K} \\
& =\int_{-\pi}^{\pi} \frac{\mathrm{d} \psi}{2 \pi} \exp (-\mathrm{i} \psi k+\phi c(\cos \psi-1)) \\
& =\mathrm{e}^{-\phi c} \int_{-\pi}^{\pi} \frac{\mathrm{d} \psi}{2 \pi} \mathrm{e}^{-\mathrm{i} \psi k} \sum_{n \geqslant 0} \frac{(\phi c)^{n}}{2^{n} n !}\left(\mathrm{e}^{\mathrm{i} \psi}+\mathrm{e}^{-\mathrm{i} \psi}\right)^{n} \\
& =\mathrm{e}^{-\phi c} \int_{-\pi}^{\pi} \frac{\mathrm{d} \psi}{2 \pi} \mathrm{e}^{-\mathrm{i} \psi k} \sum_{n \geqslant 0} \frac{(\phi c)^{n}}{2^{n} n !} \sum_{l \leqslant n} \frac{n !}{l !(n-l) !} \mathrm{e}^{-\mathrm{i} \psi(k-n+2 l)} \\
& =\mathrm{e}^{-\phi c} \sum_{n \geqslant 0} \sum_{l \leqslant n}\left(\frac{\phi c}{2}\right)^{n} \frac{1}{l !(n-l) !} \delta_{n, k+2 l} \\
& =\mathrm{e}^{-\phi c} \sum_{l \geqslant 0}\left(\frac{\phi c}{2}\right)^{k+2 l} \frac{1}{l !(k+l) !}=\mathrm{e}^{-\phi c} \mathcal{I}_{k}(\phi c)
\end{aligned}
$$

where $\mathcal{I}_{k}(x)$ is the $k$ th modified Bessel function of the first kind. These modified Bessel functions obey

$$
\begin{aligned}
& 2 \frac{k}{x} \mathcal{I}_{k}(x)=\mathcal{I}_{k-1}(x)-\mathcal{I}_{k+1}(x), \\
& 2 \frac{\mathrm{d}}{\mathrm{d} x} \mathcal{I}_{k}(x)=\mathcal{I}_{k-1}(x)+\mathcal{I}_{k+1}(x) .
\end{aligned}
$$


The first identity leads to a useful recursive equation for $\pi(k \mid \phi)$, and the second identity simplifies our calculation of derivatives of $\pi(k \mid \phi)$ with respect to $\phi$, respectively:

$$
\begin{aligned}
& \pi(k-1 \mid \phi)-\pi(k+1 \mid \phi)-2 \pi(k \mid \phi) \frac{k}{\phi c}=0, \\
& \frac{\mathrm{d}}{\mathrm{d} \phi} \pi(k \mid \phi)=c\left(\frac{1}{2} \pi(k-1 \mid \phi)+\frac{1}{2} \pi(k+1 \mid \phi)-\pi(k \mid \phi)\right) .
\end{aligned}
$$

\subsection{Retrieval in the zero noise limit}

To emphasize the dependence of the recall overlap on $\phi$, viz. the relative storage load, we will from now on write $m \rightarrow m_{\phi}$. With the abbreviation $\langle g(k)\rangle_{k}=\sum_{k} \pi(k \mid \phi) g(k)$, and using (41) and the symmetry of $\pi(k \mid \phi)$, we can transfer our equation (37) into a more convenient form:

$$
\begin{aligned}
m_{\phi} & =\frac{1}{2}\left\langle\left[\tanh \left(\beta c m_{\phi}(1+k)\right)+\tanh \left(\beta c m_{\phi}(1-k)\right)\right]\right\rangle_{k} \\
& =\frac{1}{2} \sum_{k \in \mathbb{Z}}[\pi(k-1 \mid \phi)-\pi(k+1 \mid \phi)] \tanh \left(\beta c m_{\phi} k\right)=\frac{1}{\phi c}\left\langle k \tanh \left(\beta c m_{\phi} k\right)\right\rangle_{k} .
\end{aligned}
$$

In the zero noise limit $\beta \rightarrow \infty$, where $\tanh (\beta y) \rightarrow \operatorname{sgn}(y)$, this reduces to $m_{\phi}=\frac{1}{\phi c}\langle|k|\rangle_{k}$, or, equivalently,

$$
\begin{aligned}
m_{\phi} & =\lim _{\beta \rightarrow \infty}\langle\tanh (\beta c m(1+k))\rangle_{k}=\langle\operatorname{sign}(1+k)\rangle_{k} \\
& =\sum_{k>-1} \pi(k)-\sum_{k<-1} \pi(k)=\pi(0 \mid \phi)+\pi(1 \mid \phi) .
\end{aligned}
$$

Hence we always have a non-zero rescaled magnetization, for any relative storage load $\phi$. To determine for which value of $\phi$ this state is most stable, we have to insert this solution into the zero temperature formula for the free energy and find the minimum with respect to $\phi$. Here, with $m_{\mu}=m_{\phi}$ for all $\mu \leqslant K=\phi N_{T}^{\gamma}$ and $m_{\mu}=0$ for $\mu>K$, the free energy (24) takes asymptotically the form

$$
f(\beta)=\frac{1}{2} c^{2} \phi m_{\phi}^{2}-\frac{1}{\beta}\left\langle\log \cosh \left(\beta c m_{\phi} k\right)\right\rangle_{k}-\frac{1}{\beta} \log 2 .
$$

So for $\beta \rightarrow \infty$, and using our above identity $\langle|k|\rangle_{k}=\phi c m$, we find that the energy density is

$$
\begin{aligned}
u(\phi) & =\lim _{\beta \rightarrow \infty} f(\beta)=\frac{1}{2} c^{2} \phi m_{\phi}^{2}-c m\langle|k|\rangle_{k}=-\frac{1}{2} c^{2} \phi m_{\phi}^{2} \\
& =-\frac{1}{2} c^{2} \phi(\pi(0 \mid \phi)+\pi(1 \mid \phi))^{2} .
\end{aligned}
$$

To see how this depends on $\phi$ we may use (42), and find

$$
\begin{aligned}
\frac{1}{c^{2}} \frac{\mathrm{d}}{\mathrm{d} \phi} u(\phi) & =-\frac{1}{2} m_{\phi}^{2}-\phi m_{\phi} \frac{\mathrm{d}}{\mathrm{d} \phi}(\pi(0 \mid \phi)+\pi(1 \mid \phi)) \\
& =-\frac{1}{2} m_{\phi}^{2}-\phi c m_{\phi}\left(-\frac{1}{2} \pi(0 \mid \phi)+\frac{1}{2} \pi(2 \mid \phi)\right)=-\frac{1}{2} m_{\phi}^{2}+m_{\phi} \pi(1 \mid \phi) \\
& =-\frac{1}{2} m_{\phi}\left(m_{\phi}-2 \pi(1 \mid \phi)\right)=-\frac{1}{2} m_{\phi}(\pi(0 \mid \phi)-\pi(1 \mid \phi))<0 .
\end{aligned}
$$


The energy density $u(\phi)$ is apparently a decreasing function of $\phi$, which reaches its minimum when the number of condensed patterns is maximal, at $\phi=\alpha$. However, the amplitude of each recalled pattern will also decrease for larger values of $\phi$ :

$$
\frac{\mathrm{d}}{\mathrm{d} \phi} m_{\phi}=\frac{\mathrm{d}}{\mathrm{d} \phi} \pi(0 \mid \phi)+\frac{\mathrm{d}}{\mathrm{d} \phi} \pi(1 \mid \phi)=-\pi(1 \mid \phi) / \phi<0 .
$$

Hence $m_{\phi}$ starts at $m_{0}=1$, due to $\pi(k \mid 0)=\delta_{k, 0}$, and then decays monotonically with $\phi$. Moreover, it follows from $\langle|k|\rangle_{k}^{2} \leqslant\left\langle k^{2}\right\rangle_{k}=\left\langle\sum_{\mu \leqslant K}\left(\xi^{\mu}\right)^{2}\right\rangle_{\xi}=\phi c$ that

$$
m_{\phi}=\langle|k|\rangle_{k} / \phi c \leqslant 1 / \sqrt{\phi c}, \quad u(\phi)=-\frac{1}{2} c^{2} \phi m_{\phi}^{2} \geqslant-\frac{1}{2} c .
$$

If we increase the number of condensed patterns, the corresponding magnetizations decrease in such a way that the energy density remains finite.

\subsection{Retrieval at non-zero noise levels}

To find the critical noise level (if any) where pattern recall sets in, we return to equation (25), which for $(\tau, \theta)=(1-\gamma, 0)$ and written in vector notation becomes

$$
\boldsymbol{m}=\frac{N_{T}^{\gamma}}{c}\langle\boldsymbol{\xi} \tanh (\beta c \xi \cdot \boldsymbol{m})\rangle_{\xi}
$$

We take the inner product on both sides with $\boldsymbol{m}$ and obtain a simple inequality:

$$
\begin{aligned}
\boldsymbol{m}^{2} & =\frac{N_{T}^{\gamma}}{c}\langle(\boldsymbol{\xi} \cdot \boldsymbol{m}) \tanh (\beta c \boldsymbol{\xi} \cdot \boldsymbol{m})\rangle_{\xi} \\
& =\beta N_{T}^{\gamma}\left\langle(\boldsymbol{\xi} \cdot \boldsymbol{m})^{2} \int_{0}^{1} \mathrm{~d} x\left[1-\tanh ^{2}(\beta c x \boldsymbol{\xi} \cdot \boldsymbol{m})\right]\right\rangle_{\xi} \\
& \leqslant \beta N_{T}^{\gamma}\left\langle(\boldsymbol{\xi} \cdot \boldsymbol{m})^{2}\right\rangle_{\xi}=\beta c \boldsymbol{m}^{2} .
\end{aligned}
$$

Since $\boldsymbol{m}^{2}(1-\beta c) \leqslant 0$, we are sure that $\boldsymbol{m}=0$ for $\beta c \leqslant 1$. At $\beta c=1$ nontrivial solutions of the previously studied symmetric type are found to bifurcate continuously from the trivial solution. This can be seen by expanding the amplitude equation (43) for small $m$ :

$$
\begin{aligned}
m_{\phi} & =\frac{1}{\phi c}\left\langle k \tanh \left(\beta c m_{\phi} k\right)\right\rangle_{k} \\
& =\beta c m_{\phi}-\frac{1}{3} \beta^{3} c^{2} m_{\phi}^{3}\left\langle k^{4}\right\rangle_{k} / \phi+O\left(m_{\phi}^{4}\right) .
\end{aligned}
$$

This shows that the symmetric solutions indeed bifurcate via a second-order transition, at the $\phi$-independent critical temperature $T_{c}=c$, with amplitude $m_{\phi} \propto(\beta c-1)^{\frac{1}{2}}$ as $\beta c \rightarrow 1$. All the above predictions are confirmed by the results of numerical simulations, and by solving the order parameter equations and calculating the free energy numerically, see figure 9.

We can now summarize the phase diagram in terms of the scaling exponents $(\gamma, \delta)$. The number of stored patterns is $N_{B}=\alpha N_{T}^{\delta}$, of which $K=\phi N_{T}^{\delta^{\prime}}$ can be recalled simultaneously, with $\delta^{\prime}=\min (\gamma, \delta)$ :

$\delta<\gamma: \phi_{\max }=\alpha$, all stored patterns recalled simultaneously, with Curie-Weiss overlap $m$

$\delta=\gamma: \phi_{\max }=\alpha$, all stored patterns recalled simultaneously, with reduced but finite $m$

$\delta>\gamma: \phi_{\max }=\infty$, at most $\phi N_{T}^{\gamma}$ patterns recalled simultaneously, with $\phi \rightarrow \infty$ and $m_{\phi} \rightarrow 0$. 

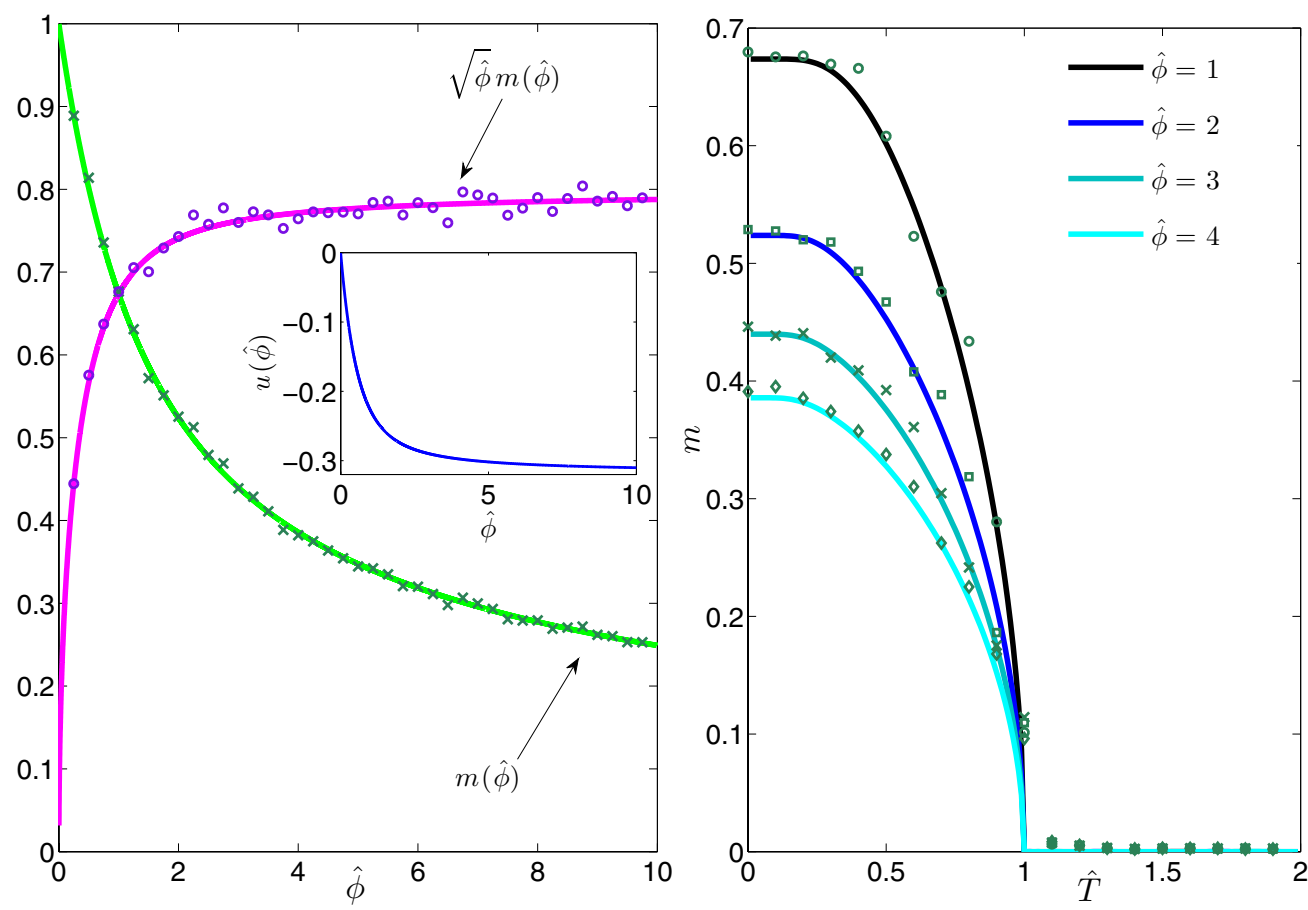

Figure 9. Left: energy density $u$ versus the relative fraction of retrieved patterns, in terms of $\hat{\phi}=c \phi$ and $\hat{T}=T / c=1 / \beta c$. The minimum energy density is reached when $\hat{\phi}$ is maximal, i.e. when all stored patterns are simultaneously retrieved, but with decreasing amplitude for each. Right: critical noise levels for different values of $\hat{\phi}$, confirming that $\hat{T}_{c}^{-1}=\hat{\beta}_{c}=1$, independently of $\hat{\phi}$. In both the panels, solid lines represent our theoretical predictions, while symbols represent data from numerical simulations on systems with $N_{T}=5 \times 10^{4}, \gamma=\delta=0.45, c=2$ and with standard sequential Glauber dynamics.

\section{High storage regime in extremely diluted connectivity: absence of retrieval}

Let us finally consider the same network, composed of $N_{T}$ T-clones $\left(\sigma_{i}, i=1, \ldots, N_{T}\right)$ and $N_{B}$ B-clones $\left(b_{\mu}, \mu=1, \ldots, N_{B}\right)$, but now at high storage load:

$$
\lim _{N_{T} \rightarrow \infty} N_{B} / N_{T}=\alpha, \quad \alpha>0 .
$$

The effective interaction between T-cells is still described by the Hamiltonian (19), and the cytokine variables $\xi_{i}^{\mu} \in\{0, \pm 1\}$ are generated from (20), but now we focus on the extremely diluted regime for the B-T network, i.e. $\gamma<1$. Again we must choose $\tau$ such that the Hamiltonian will be of order $N_{T}$. Heuristically, since the number of non-zero entries $\mathcal{N}_{\mathrm{nz}}$ in a typical pattern $\left(\xi_{1}^{\mu}, \ldots, \xi_{N_{T}}^{\mu}\right)$ scales as $O\left(N_{T}^{1-\gamma}\right)$, the number of patterns with non-overlapping entries (i.e. those we expect to recall) will scale as $O\left(N_{T} / \mathcal{N}_{\mathrm{nz}}\right)=O\left(N_{T}^{\gamma}\right)$. The contribution from $K=O\left(N_{T}^{\gamma}\right)$ such condensed patterns to the Hamiltonian would then scale as

$$
\mathcal{H}_{C} \sim N_{T}^{-\tau} \sum_{\mu=1}^{K}\left(\sum_{i=1}^{N_{T}} \xi_{i}^{\mu} \sigma_{i}\right)^{2} \sim N_{T}^{-\tau} K \mathcal{N}_{\mathrm{nz}}^{2} \sim N_{T}^{-\tau} N_{T}^{\gamma} N_{T}^{2(1-\gamma)} \sim N_{T}^{2-\gamma-\tau}
$$


The non-condensed patterns, of which there are $N_{\mathrm{nc}}=N_{B}-N_{c} \sim N_{B}=O\left(N_{T}\right)$, are expected to contribute

$\mathcal{H}_{\mathrm{NC}} \sim N_{T}^{-\tau} \sum_{\mu=1}^{N_{\mathrm{nc}}}\left(\sum_{i=1}^{N_{T}} \xi_{i}^{\mu} \sigma_{i}\right)^{2} \sim N_{T}^{-\tau} N_{\mathrm{nc}}{\sqrt{\mathcal{N}_{\mathrm{nz}}}}^{2} \sim N_{T}^{-\tau} N_{T} N_{T}^{1-\gamma} \sim N_{T}^{2-\gamma-\tau}$.

Thus, we expect to have an extensive Hamiltonian for $\tau=1-\gamma$.

\subsection{Replica-symmetric theory}

In the scaling regime $N_{B}=\alpha N_{T}$ we can no longer use saddle-point arguments directly in the calculation of the free energy. Instead we calculate the free energy for typical cytokine realizations, i.e. the average

$$
\bar{f}=-\lim _{N_{T} \rightarrow \infty} \frac{1}{\beta N_{T}} \overline{\log Z_{N_{T}}(\beta, \xi)}
$$

Here $\cdots$ indicates averaging over all $\left\{\xi_{i}^{\mu}\right\}$, according to the measure (20). The average over cytokine variables is done with the replica method, for $K=O\left(N_{T}^{\gamma}\right)$; full details are given in appendix B. We solve the model at the replica symmetric (RS) level, which implies the assumption that the system has at most a finite number of ergodic sectors for $N_{T} \rightarrow \infty$, giving

$$
\begin{aligned}
\beta \bar{f}_{\mathrm{RS}}=\lim _{N_{T} \rightarrow \infty} & \operatorname{extr}_{\boldsymbol{m}, q, r} \beta \hat{f}_{\mathrm{RS}}(\boldsymbol{m}, q, r) \\
\beta \hat{f}_{\mathrm{RS}}(\boldsymbol{m}, q, r) & =-\log 2+\frac{1}{2} \alpha r(\beta c)^{2}(1-q) \\
& +\frac{\beta c^{2}}{2 N_{T}^{\gamma}} \boldsymbol{m}^{2}-\frac{\alpha}{2}\left(\frac{\beta c q}{1-\beta c(1-q)}-\log [1-\beta c(1-q)]\right) \\
& -\left\langle\int \mathrm{D} z \log \cosh [\beta c(\boldsymbol{m} \cdot \boldsymbol{\xi}+z \sqrt{\alpha r})]\right\rangle_{\xi}
\end{aligned}
$$

in which $\boldsymbol{m}=\left(m_{1}, \ldots, m_{K}\right)$ denotes the vector of $K=\phi N_{T}^{\gamma}$ condensed (i.e. potentially recalled) patterns, $\xi=\left(\xi^{1}, \ldots, \xi^{K}\right)$, and $\mathrm{D} z=(2 \pi)^{-1 / 2} \mathrm{e}^{-z^{2} / 2} \mathrm{~d} z$. As in the analysis of standard Hopfield networks, this involves the Edward-Anderson spin-glass order parameter $q$ $[35,51]$ and the Amit-Gutfreund-Sompolinsky uncondensed-noise order parameter $r[35,51]$. We obtain self-consistent equations for the remaining RS order parameters $(m, q, r)$ simply by extremizing $\hat{f}_{\mathrm{RS}}(\boldsymbol{m}, q, r)$, which leads to

$$
\begin{aligned}
& m^{\mu}=\frac{N_{T}^{\gamma}}{c}\left\langle\xi^{\mu} \int \mathrm{D} z \tanh [\beta c(\boldsymbol{m} \cdot \boldsymbol{\xi}+z \sqrt{\alpha r})]\right\rangle_{\xi}, \\
& q=\left\langle\int \mathrm{D} z \tanh ^{2}[\beta c(\boldsymbol{m} \cdot \xi+z \sqrt{\alpha r})]\right\rangle_{\xi}, \\
& r=\frac{q}{[1-\beta c(1-q)]^{2}} .
\end{aligned}
$$

As before we deal with the equation for $m^{\mu}$ by using the identity $\xi^{\mu} \tanh (A)=\tanh \left(\xi^{\mu} A\right)$ (since $\xi^{\mu} \in\{-1,0,1\}$ ) and by separating the term $m^{\mu} \xi^{\mu}$ from the sum $\boldsymbol{m} \cdot \boldsymbol{\xi}$ :

$$
\begin{aligned}
m^{\mu} & =\frac{N_{T}^{\gamma}}{c}\left\langle\int \mathrm{D} z \tanh \left[\beta c\left(m^{\mu}\left(\xi^{\mu}\right)^{2}+\sum_{\nu \neq \mu \leqslant K} m^{\nu} \xi^{\nu} \xi^{\mu}+z \xi^{\mu} \sqrt{\alpha r}\right)\right]\right\rangle_{\xi} \\
& =\left\langle\int \mathrm{D} z \tanh \left[\beta c\left(m^{\mu}+\sum_{\nu \neq \mu \leqslant K} m^{\nu} \xi^{\nu}+z \sqrt{\alpha r}\right)\right]\right\rangle_{\xi}
\end{aligned}
$$




$$
=\left\langle\int \mathrm{D} z \tanh \left[\beta c\left(m^{\mu}+\sum_{\nu=1}^{K} m^{\nu} \xi^{\nu}+z \sqrt{\alpha r}\right)\right]\right\rangle_{\xi}+O\left(N^{-\gamma}\right) .
$$

Again we see that for $N_{T} \rightarrow \infty$ we will only retain solutions with $m^{\mu} \in\{-m, 0, m\}$ for all $\mu \leqslant K$. Given the trivial sign and pattern label permutation invariances, we can without loss of generality consider only non-negative magnetizations, and look for solutions where $m^{\mu}=m$ for $\mu=1 \leqslant K$ and zero otherwise. We then find

$$
m=\sum_{k=-\infty}^{\infty} \pi(k) \int \mathrm{D} z \tanh [\beta c(m+m k+z \sqrt{\alpha r})]
$$

with $\pi(k)$ given in (39). We can now use the manipulations employed in the previous section, to find

$$
\begin{aligned}
m & =\left\langle\frac{k}{\phi} \int \mathrm{D} z \tanh [\beta c(m k+z \sqrt{\alpha r})]\right\rangle_{k} \\
q & =\left\langle\int \mathrm{D} z \tanh ^{2}[\beta c(m k+z \sqrt{\alpha r})]\right\rangle_{k}, \\
r & =\frac{q}{[1-\beta c(1-q)]^{2}} .
\end{aligned}
$$

The corresponding free energy assumes the form

$$
\begin{aligned}
\beta \hat{f}_{\mathrm{RS}}(m, q, r)= & -\log 2+\frac{1}{2} \alpha r(\beta c)^{2}(1-q)+\frac{1}{2} \beta c^{2} \phi m^{2} \\
& -\frac{\alpha}{2}\left(\frac{\beta c q}{1-\beta c(1-q)}-\log [1-\beta c(1-q)]\right) \\
& -\left\langle\int \mathrm{D} z \log \cosh [\beta c(m k+z \sqrt{\alpha r})]\right\rangle_{k} .
\end{aligned}
$$

Note that we recover the equations of the medium storage regime simply by putting $\alpha=0$.

\subsection{The zero noise limit}

We now show that in the high storage case the system behaves as a spin-glass, even in the zero temperature limit $\beta \rightarrow \infty$ where the retrieval capability should be largest. From (63) we deduce that $q \rightarrow 1$ in the zero noise limit, while the quantity $C=\beta c(1-q)$ remains finite. Let us first send $\beta \rightarrow \infty$ in equation (62):

$$
m=\left\langle\frac{k}{\phi} \int \mathrm{D} z \operatorname{sgn}\left[m k+\frac{z \sqrt{\alpha}}{1-C}\right]\right\rangle_{k}=\left\langle\frac{k}{\phi} \operatorname{Erf}\left(\frac{m k(1-C)}{\sqrt{2 \alpha}}\right)\right\rangle_{k},
$$

with the error integral $\operatorname{Erf}(x)=(2 / \sqrt{\pi}) \int_{0}^{x} \mathrm{~d} t \mathrm{e}^{-t^{2}}$. A second equation for the pair $(m, C)$ follows from (63):

$$
\begin{aligned}
C & =\lim _{\beta \rightarrow \infty} \beta c\left\langle 1-\int \mathrm{D} z \tanh ^{2}[\beta c(m k+z \sqrt{\alpha r})]\right\rangle_{k} \\
& =\lim _{\beta \rightarrow \infty} \frac{\partial}{\partial m}\left\langle\frac{1}{k} \int \mathrm{D} z \tanh \left[\beta c\left(m k+\frac{z \sqrt{\alpha q}}{1-C}\right)\right]\right\rangle_{k}, \\
& =\frac{\partial}{\partial m}\left\langle\frac{1}{k} \operatorname{Erf}\left(\frac{m k(1-C)}{\sqrt{2 \alpha}}\right)\right\rangle_{k} \\
& =\sqrt{\frac{2}{\alpha \pi}}(1-C)\left\langle\exp \left(-\frac{m^{2} k^{2}(1-C)^{2}}{2 \alpha}\right)\right\rangle_{k} .
\end{aligned}
$$



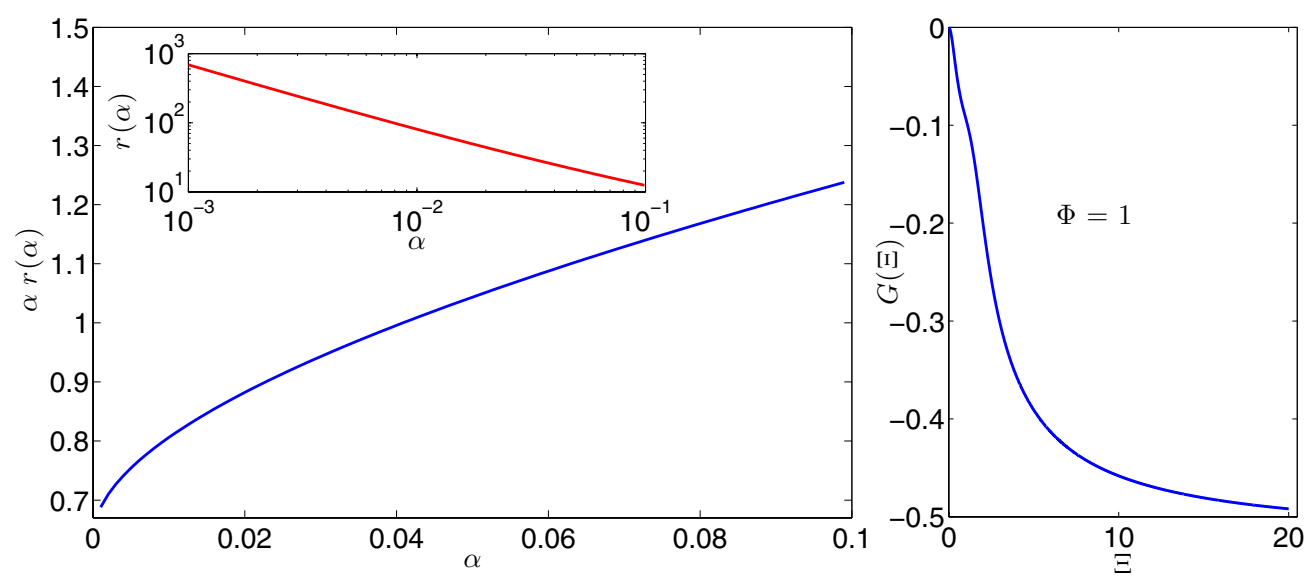

Figure 10. Left panel: behaviour of $\alpha r(\alpha)$ versus $\alpha$ in the spin-glass state (the inset shows only $r(\alpha)$ versus $\alpha)$, as calculated from the RS order parameter equations. This shows that $r(\alpha)$ goes to infinity as $\alpha$ approaches zero, such that $\alpha r(\alpha)$ remains positive; this means that the noise due to non-condensed patterns can never be neglected. Right panel: behaviour of the function $G(\Xi)$ versus $\Xi$. Since $G(\Xi)<0$ for $\alpha>0$, equation (69) cannot have a solution for $\alpha>0$, and hence no pattern recall is possible even at zero noise.

We thus have two coupled nonlinear equations (65), (66), for the two zero temperature order parameters $m$ and $C$. They can be further reduced by introducing the variable $\Xi=m(1-C) / \sqrt{2 \alpha}$, with which we obtain

$$
m=\left\langle\frac{k}{\phi} \operatorname{Erf}(k \Xi)\right\rangle_{k}
$$

and rewriting $\Xi=m(1-C) / \sqrt{2 \alpha}$ gives

$$
C=1-\frac{\sqrt{2 \alpha} \Xi}{m}=1-\sqrt{2 \alpha} \Xi\left\langle\frac{k}{\phi} \operatorname{Erf}(k \Xi)\right\rangle_{k}^{-1} .
$$

Using (66) and excluding the trivial solution $\Xi=0$ (which always exists, but represents the spin-glass state without pattern recall) we obtain after some simple algebra just a single equation, to be solved for $\Xi$ :

$$
\sqrt{2 \alpha}=G(\Xi)=\frac{1}{\Xi}\left\langle\frac{k}{\phi} \operatorname{Erf}(k \Xi)\right\rangle_{k}-\frac{2}{\sqrt{\pi}}\left\langle\mathrm{e}^{-k^{2} \Xi^{2}}\right\rangle_{k} .
$$

One easily shows that

$$
\lim _{\Xi \rightarrow 0} G(\Xi)=0, \quad \lim _{\Xi \rightarrow \infty} G(\Xi)=-\frac{2}{\sqrt{\pi}} \pi(0 \mid \phi) .
$$

In fact further analytical and numerical investigation reveals that for $\Xi>0$ the function $G(\Xi)$ is strictly negative; see figure 10 . Hence there can be no $m \neq 0$ solution for $\alpha>0$, so the system cannot recall the patterns in the present scaling regime $N_{B}=\alpha N_{T}$.

\section{Conclusions}

The immune system is a marvellous complex biological entity, able to execute reliably a number of very difficult tasks that allow living beings to survive in competitive interaction 
with a living environment. To accomplish this it relies on a huge ensemble of functions and agents. In particular, the adaptive part of the immune system relies on a broad ensemble of cells, e.g. B and T lymphocytes, and of chemical messengers, e.g. antibodies and cytokines. As for lymphocytes, one can distinguish between an 'effector branch', consisting of B-cells and killer T-cells, and an 'organizational branch', which coordinates the operation of the effector branch and consists mainly of helper and regulator T-cells. The latter control the activity of the effector branch through a rich and continuous exchange of cytokines, which are specific chemical messengers which elicit or suppress effector actions.

From a theoretical point of view, a fascinating ability of the immune system is its simultaneous management, by helpers and suppressors, of several B-clones at once; this is a key ability, as it implies the ability to defend the host from simultaneous attacks by several pathogens. Indeed, we investigated this ability in the present study, as an emergent, collective, feature of a spin-glass model of the immune network, that describes the adaptive response performed by B-cells under the coordination of helpers and suppressors. In particular, the focus of this paper is on the ability of the T-cells to coordinate an extensive number of B-soldiers, by fine-tuning the load of clones and the degree of dilution in the network.

As a starting point we assumed symmetric interactions among clones. Although this is a first necessary step in order to achieve a full equilibrium theory, this symmetry is not a biological requisite. However, we note that, while cytokines have a directionality and form gradients, the linkage between helpers and B-cells via the CD40 arm is intrinsically symmetric, thus conferring some sort of stability. In neural network models it was found that robustness properties regarding retrieval, and in general most of the emergent behaviour, largely survived in off-equilibrium extensions. We expect that the same will be true for immune networks.

It is worth considering the parallel processing capability also from a slightly different perspective. Beyond the interest in multiple clonal expansions (which, in our language, is achieved through signalling by +1 cytokines), the quiescence signals that are sent to the Bclones that are not expanding (which, in our language, is achieved through signalling by -1 cytokines) is fundamental for homeostasis. In fact, B-cells that are not receiving a significant amount of signals undergo a depauperation process called 'anergy' $[45,46]$ and eventually die. Hence, in the present multi-tasking network, the capability of signalling simultaneously to all clones is fundamental, and with implications beyond solely the management of simultaneous clonal expansions; we emphasize that within our approach this is achieved in a rather natural way.

We first assumed that the number $N_{B}$ of B-cells scales with the number $N_{T}$ of T-cells as $N_{B}=\alpha N_{T}^{\delta}$, with $\delta<1$, and we modelled the interaction between B-cells and T-cells by means of an extremely diluted bipartite spin-glass where the former are addressed only by a subset of T-cells whose cardinality scales like $N_{T}^{1-\gamma}$, with $\gamma \leqslant 1$. We proved that this system is thermodynamically equivalent to a diluted monopartite graph, whose topological properties are shown to depend crucially on the parameters $\gamma$ and $\delta$. In particular, when $\gamma \geqslant \delta$ the graph is fragmented into multiple disconnected components, each forming a clique or a collection of cliques typically connected via a bridge. Each clique corresponds to a pattern and this kind of arrangement easily allows for the simultaneous recall of multiple patterns. On the other hand, when $\gamma<\delta$, the effective network can exhibit a giant component, which prevents the system from simultaneous pattern recall. These results on the topology of the immune network are then approached from a statistical mechanics angle: we analyse the operation of the system as an effective equilibrated stochastic process of interacting helper cells. We find that for $\gamma>\delta$ the network is able to retrieve perfectly all the stored patterns simultaneously, in perfect agreement with the topology-based prediction. When the load increases, i.e. when $N_{B}$ becomes larger (so the exponent $\delta$ is increased), overlaps among bit entries of the 'cytokine 
patterns' to be recalled become more and more frequent, and this gives rise to a new source of non-Gaussian interference noise that is non-negligible for $\gamma \leqslant \delta$. If $\gamma=\delta$ the system is still able to retrieve all the patterns, but with a decreasing recall overlap.

Since the numbers of cells, i.e. $N_{T}$ and $N_{B}$, are fluctuating quantities, their ratio $\alpha$ is not constant in real networks. It is therefore important that the system's phase diagram exhibits significant regions of $\alpha$ values where the system would operate adequately. Deriving this phase diagram was our goal.

In the high storage case, for $\delta=1$, the network starts to feel also the Gaussian noise due to non-condensed patterns, and this is found to destroy the retrieval states. Here the system behaves as a spin-glass, from which we deduce that an extremely diluted B-H network (i.e. one with $\gamma<1$ ) is insufficiently diluted to sustain a high pattern load. Our predictions and results are tested against numerical simulations wherever possible, and we consistently find perfect agreement.

Despite the fact that it is experimentally well established that helpers are much more numerous than B-cells, their relative sizes are still comparable in a statistical mechanical sense. The biological interest lies in the high storage regime, where the maximum number of pathogens can be fought simultaneously. From the present study we now know that to bypass the spin-glass structure of the phase space at this load level, a projection of the model into a finite-connectivity topology $(\gamma=1)$ is required. This, remarkably, is also in agreement with the biological picture of highly selective touch-interactions among B- and T-cells.

It is both welcome and encouraging that both biological data and statistical mechanical theory have now converged to the same suggestion: that the most efficient and biologically most plausible operation regime is likely to be that of finite connectivity for the effective helper-helper immune network. This must therefore be the direction of the next stage of our research programme.

\section{Acknowledgments}

EA, AB and DT acknowledge the FIRB grant RBFR08EKEV and Sapienza Universitá di Roma for financial support. ACCC is grateful for support from the Biotechnology and Biological Sciences Research Council (BBSRC) of the UK. DT would like to thank King's College London for hospitality.

\section{Appendix A. Topological properties}

\section{A.1. Rigorous calculation of link probability}

We consider the bipartite graph $\mathcal{B}$, and denote with $\rho_{i}$ the number of links stemming from node $i \in V_{T}$. Note that $\rho_{i}$ also gives the number of non-null entries in the string $\left(\xi_{i}^{1}, \ldots, \xi_{i}^{N_{B}}\right)$ processed at node $i$, that is

$$
\rho_{i}=\sum_{\mu=1}^{N_{B}}\left|\xi_{i}^{\mu}\right|
$$

All entries $\xi_{i}^{\mu}$ are i.i.d. variables (5), so for each node the number $\rho_{i}$ is distributed according to

$$
P\left(\rho \mid N_{B}, N_{T}, \gamma, c\right)=\left(\begin{array}{c}
N_{B} \\
\rho
\end{array}\right)\left(\frac{c}{2 N_{T}^{\gamma}}\right)^{\rho}\left(1-\frac{c}{2 N_{T}^{\gamma}}\right)^{N_{B}-\rho}
$$


When considering two distinct nodes $i, j \in V_{T}$, the number $\ell$ of shared nearest-neighbours corresponds to the number of non-null matchings between the related strings, and this is distributed according to

$P\left(\ell \mid \rho_{i}, \rho_{j}, N_{B}\right)=\frac{N_{B} !}{\left(N_{B}+\ell-\rho_{i}-\rho_{j}\right) !\left(\rho_{i}-\ell\right) !\left(\rho_{j}-\ell\right) ! \ell !}\left[\left(\begin{array}{c}N_{B} \\ \rho_{i}\end{array}\right)\left(\begin{array}{c}N_{B} \\ \rho_{j}\end{array}\right)\right]^{-1}$.

The average $\langle\ell\rangle_{\rho_{i}, \rho_{j}}$ then follows as

$$
\langle\ell\rangle_{\rho_{i}, \rho_{j}}=\rho_{i} \rho_{j} / N_{B}
$$

By further averaging over $P\left(\rho \mid N_{B}, N_{T}, c, \gamma\right)$ we get

$$
\langle\ell\rangle=\langle\rho\rangle^{2} / N_{B}
$$

Fluctuations scale as $\left\langle\ell^{2}\right\rangle-\langle\ell\rangle^{2} \sim\langle\ell\rangle^{2}$, where, from the distribution above, $\langle\rho\rangle=c N_{B} / 2 N_{T}^{\gamma}$. Upon choosing $N_{B}=\alpha N_{T}^{\delta}$ we then get $\langle\ell\rangle \sim N_{T}^{\delta-2 \gamma}$, which vanishes if $2 \gamma>\delta$. Two strings of any two nodes apparently do not display significant matching, so there is no link between them, consistent with the results of section 3.

On the other hand, if $2 \gamma<\delta$ so that $\langle\ell\rangle \gg 1$, we can approximate $P\left(J \mid N_{B}, N_{T}, \gamma, c\right)$ (the probability of two randomly drawn nodes in the effective $N_{T}$-node graph having a link $J$ ) with $P\left(J \mid\langle\ell\rangle, N_{T}, \gamma, c\right)$ : the probability that a random walk of length $N_{B}$ with a waiting probability $p_{w}$ ends at distance $J$ from the origin is approximated by the probability that a simple random walk of length $\langle\ell\rangle$ ends at the same distance (with proper normalization to account for parity features). In particular,

$$
P\left(J=0 \mid N_{B}, N_{T}, \gamma, c\right) \approx\left(\begin{array}{c}
\langle\ell\rangle \\
\langle\ell\rangle / 2
\end{array}\right) 2^{-\langle\ell\rangle} \approx \sqrt{2 / \pi\langle\ell\rangle}
$$

(using Stirling's formula in the last step). The expected link probability between two nodes follows as

$P\left(J \neq 0 \mid N_{B}, N_{T}, \gamma, c\right)=1-P\left(J=0 \mid N_{B}, N_{T}, \gamma, c\right) \approx 1-\sqrt{\frac{2}{\pi \alpha}} \frac{N_{T}^{\gamma-\delta / 2}}{c}$.

It is easy to see that when $2 \gamma=\delta$ the link probability is finite and smaller than 1 , while when $2 \gamma<\delta$ it converges to 1 in the thermodynamic limit, consistent with the results of section 3 .

\section{A.2. Generating function approach to percolation in the bipartite graph}

Let us consider a bipartite graph $\mathcal{B}$, made of two sets of nodes $V_{T}$ (of size $N_{T}$ ) and $V_{B}$ (of size $N_{B}$ ), with both sizes diverging. The degree distribution for the two parts are $p_{k}$ and $q_{k}$, respectively, with $\sum_{k} p_{k} k=\mu$ and $\sum_{k} q_{k} k=v$. Following [53], we introduce the following generating functions

$$
\begin{array}{rlrl}
f_{0}(x) & =\sum_{k=0}^{N_{T}} p_{k} x^{k}, & g_{0}(x)=\sum_{k=0}^{N_{B}} q_{k} x^{k}, \\
f_{1}(x)=\frac{1}{\mu} \frac{\mathrm{d}}{\mathrm{d} x} f_{0}(x), & g_{1}(x)=\frac{1}{v} \frac{\mathrm{d}}{\mathrm{d} x} g_{0}(x) .
\end{array}
$$

We note that $f_{1}(x)$ and $g_{1}(x)$ are the generating functions for the degree distribution of a vertex reached following a randomly chosen edge (here the degree does not include the link along which we arrived). One always has $\mu / N_{T}=v / N_{B}$, and $f_{0}(1)=g_{0}(1)=f_{1}(1)=g_{1}(1)=1$ (by construction).

Next we introduce dilution. We define the matrix $\mathbf{t}$, whose element $t_{k \ell}$ represents the probability that a directed link going from a node in part $k$ to a node in part $\ell$ exists. 
For bipartite graphs, $\mathbf{t}$ is simply a $2 \times 2$ matrix with zero diagonal entries. We can now write the generating functions for the distributions of occupied edges attached to a vertex chosen randomly as follows [53]:

$$
\begin{array}{ll}
f_{0}(x \mid \mathbf{t})=f_{0}\left(1+(x-1) t_{12}\right), & f_{1}(x \mid \mathbf{t})=f_{1}\left(1+(x-1) t_{12}\right), \\
g_{0}(x \mid \mathbf{t})=g_{0}\left(1+(x-1) t_{21}\right), & g_{1}(x \mid \mathbf{t})=g_{1}\left(1+(x-1) t_{21}\right) .
\end{array}
$$

Let us now consider a node $i \in V_{T}$, with $z_{i}$ neighbours (where $z_{i}$ is distributed according to $p_{k}$ ). Due to the dilution, only a fraction of the links that could connect to $i$ will be present. The nodes in the second part that are reached from $i$ will, in turn, have a number of links hitting some nodes in $V_{T}$. The generating function $F_{0}(x \mid \mathbf{t})$ of the distribution of nodes in the first part which are involved in both steps is

$$
\begin{aligned}
F_{0}(x \mid \mathbf{t}) & =\sum_{m=0}^{\infty} \sum_{k=m}^{\infty} p_{k}\left(\begin{array}{l}
k \\
m
\end{array}\right) t_{12}^{m}\left(1-t_{12}\right)^{k-m}\left[g_{1}(x ; \mathbf{t})\right]^{m} \\
& =f_{0}\left(g_{1}(x \mid \mathbf{t}) \mid \mathbf{t}\right)=f_{0}\left(1+\left(g_{1}(x \mid \mathbf{t})-1\right) t_{12}\right) .
\end{aligned}
$$

In fact, in the expansion of $\left[g_{1}(x ; \mathbf{t})\right]^{m}$, the coefficient of $x^{n}$ is simply the probability that $m$ randomly reached nodes are connected to a set of $n$ other nodes. If we choose an edge rather than a node we have, analogously

$$
F_{1}(x \mid \mathbf{t})=f_{1}\left(1+\left(g_{1}(x \mid \mathbf{t})-1\right) t_{12}\right) .
$$

The corresponding generating functions found upon starting with a note in the part $V_{B}$ have analogous definitions, and will be written as $G_{0}$ and $G_{1}$.

The generating function $H_{0}$ for the distribution $P(s \mid \mathbf{t})$ of the size $s$ of the components (connected sub-graphs) which one can detect is $H_{0}(x \mid \mathbf{t})=\sum_{s} P(s \mid \mathbf{t}) x^{s}$. Similarly, $H_{1}(x \mid \mathbf{t})$ will be the generating function for the size of the cluster of connected vertices that we reach by following a randomly chosen vertex. We note that in the highly diluted regimes we can exploit the fact that the probability of finding closed loops is $O\left(N_{T}^{-1}\right.$ ) (so $H_{0}$ and $H_{1}$ do not include the giant component), which allows us to write the explicit expressions

$$
H_{0}(x \mid \mathbf{t})=x F_{0}\left(H_{1}(x \mid \mathbf{t}) \mid \mathbf{t}\right), \quad H_{1}(x \mid \mathbf{t})=x F_{1}\left(H_{1}(x \mid \mathbf{t}) \mid \mathbf{t}\right) .
$$

This then gives for the average cluster size:

$$
\langle s\rangle=H_{0}^{\prime}(1 \mid \mathbf{t})=1+F_{0}^{\prime}(1 \mid \mathbf{t}) H_{1}^{\prime}(1 \mid \mathbf{t})=1-\frac{F_{0}^{\prime}(1 \mid \mathbf{t})}{1-F_{1}^{\prime}(1 \mid \mathbf{t})},
$$

where we used $H_{1}^{\prime}(1 \mid \mathbf{t})=1+F_{1}^{\prime}(1 \mid \mathbf{t}) H_{1}^{\prime}(1 \mid \mathbf{t})$. As for $F_{0}$ and $F_{1}$, recalling equations (A.12) and (A.13) we get $F_{0}^{\prime}(1 \mid \mathbf{t})=f_{0}\left(g_{1}\left(1 \mid t_{21}\right) \mid t_{12}\right) g_{1}^{\prime}\left(1 \mid t_{21}\right)=f_{0}^{\prime}\left(1 \mid t_{12}\right) g_{0}^{\prime}(1) t_{21}$, and analogous formulae for $F_{1}(1 \mid \mathbf{t})$. Therefore,

$$
\langle s\rangle=1+\frac{t_{12} t_{21} f_{0}^{\prime}(1) g_{0}^{\prime}(1)}{1-t_{12} t_{21} f_{1}^{\prime}(1) g_{1}^{\prime}(1)} .
$$

This expression diverges for

$$
t_{12} t_{21}=\frac{1}{f_{1}^{\prime}(1) g_{1}^{\prime}(1)}=\frac{\mu \nu}{\left(\sum_{j} j(j-1) p_{j}\right)\left(\sum_{k} k(k-1) q_{k}\right)}
$$

signalling the phase transition at which a giant-component first appears. Assuming $\mathbf{t}$ to be symmetric, as we are interested in equilibrium statistical-mechanical descriptions of the system, the left-hand side of the previous equations simplifies into $t^{2} \equiv t_{12}^{2}$. As for the degree distributions $p_{k}$ and $q_{k}$, the case considered in the main text is the easiest one, viz. an 


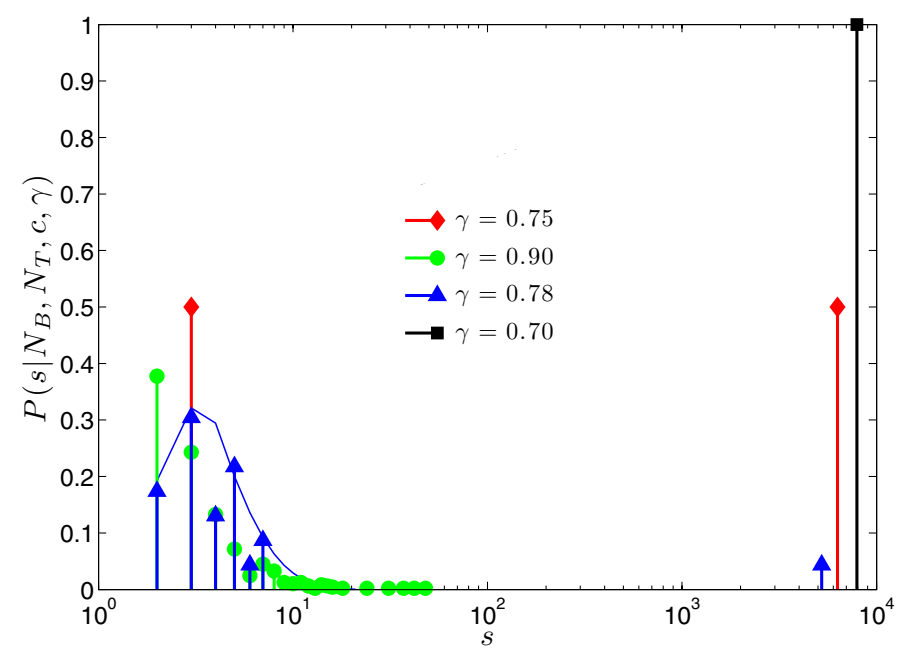

Figure A1. Distribution for the cluster size $P\left(s \mid N_{B}, N_{T}, c, \gamma\right)$ measured numerically over a simulated graph $\mathcal{G}$ with $N_{T}=10^{4}$ nodes. The parameters $\delta=1, \alpha=0.5$ and $c=1$ are kept fixed, while $\gamma$ is varied (see legend). In the interest of clarity, we plotted the analytical estimate of equations (A.20) and (A.22) only for $\gamma=0.78$.

originally fully-connected bipartite network that has been progressively diluted, in such a way that $p_{k}=\delta_{k, N_{T}-1}$ and $q_{k}=\delta_{k, N_{B}-1}$. Hence, we immediately find

$$
t^{2} \approx 1 / N_{T} N_{B}
$$

As mentioned earlier, the distribution for the size of the small components reached from a randomly chosen node $i \in V_{T}$ has generating function $H_{0}(x \mid \mathbf{t})=x F_{1}\left(H_{1}(x \mid \mathbf{t}) \mid \mathbf{t}\right)$.

The generating function approach implicitly assumes that small components are always tree-like, i.e. the probability of finding closed loops in finite components is negligible in the large system size limit. Hence, if $t=1-c / N_{T}^{\gamma}$, then $\gamma \geqslant 1$, so we are in effect considering how the system approaches the percolation threshold from the underpercolated regime. Therefore, $H_{1}(x \mid \mathbf{t}) \approx x$ in such a way that

$$
F_{1}(x \mid \mathbf{t})=\left\{1-t+t[1+(x-1) t]^{N_{B}-2}\right\}^{N_{T}-2} .
$$

Upon substituting (A.19) into the expression for $H_{0}(x \mid \mathbf{t})$, followed by a numerical inverse Laplace transform, we obtain clear evidence that, when $s$ is small, the leading term for $P(s \mid \mathbf{t})$ decreases exponentially with $s$. This prediction is confirmed by numerical simulations, see the right panel of figure 4. More generally, the distribution of component sizes can be written as

$P\left(s \mid N_{B}, N_{T}, c, \gamma\right)=\left(\begin{array}{c}N_{T} \\ s\end{array}\right) \sum_{l=1}^{N_{B}}\left(\begin{array}{c}N_{B} \\ l\end{array}\right)(1-p)^{s\left(N_{B}-l\right)}(1-p)^{l\left(N_{T}-s\right)} C(l, s)$,

where we accounted for the probability of choosing a component $\left(\subseteq V_{T}\right)$ of size $s$ linked through, overall, $l$ nodes $\left(\subseteq V_{B}\right)$, and for the probability that this sub-graph is disconnected from the remaining nodes; $C(l, s)$ is the probability that a subgraph made of $s+l$ elements is connected. Now, the $s$ nodes $\in V_{T}$ can be partitioned into sub-graphs $\left\{s_{1}, s_{2}, \ldots, s_{l}\right\}$, where $s_{k}$ includes all nodes linked to exactly $k$ nodes among the $l$ selected. Therefore, we can write

$$
C(l, s)=\sum_{s_{1}, \ldots, s_{l}} \frac{s ! \delta_{s, \sum_{k}\left|s_{k}\right|}}{\prod_{k=1}^{l}\left|s_{k}\right| !} \prod_{k=1}^{l}\left[p^{k}(1-p)^{l-k}\right]^{\left|s_{k}\right|} .
$$


A simple upper bound for $C(l, s)$ follows by imposing that all $s+l$ nodes are connected to at least one node

$$
C(l, s) \leqslant \tilde{C}(l, s)=\left[1-(1-p)^{l}\right]^{s}\left[1-(1-p)^{s}\right]^{l} .
$$

This does not imply that the whole sub-graph is connected, but the bound is a good approximation when the link probability is either low or high. Using this approximation and the expression $p=c / N_{T}^{\gamma}$, we find that: for relatively small $\gamma$ only the case $s \sim O\left(N_{T}\right)$ and $l \sim O\left(N_{B}\right)$ has non-vanishing probability, for relatively large $\gamma$ only the case with $s$ and $l$ finite has non-vanishing probability, and for intermediate values of $\gamma$ both these extreme cases are possible. In figure A1 we show a comparison between analytical and numerical results.

\section{Appendix B. Free energy evaluation using the replica method}

In this appendix we calculate the free energy per spin of the system characterised by the Hamiltonian (19), within the replica-symmetric (RS) ansatz, for the scaling regime $N_{B}=\alpha N_{T}$. Let us start by introducing the partition function $Z_{N_{T}}(\beta, \xi)$ and the disorder-averaged free $\operatorname{energy} \bar{f}$ :

$$
\begin{aligned}
& Z_{N_{T}}(\beta, \xi)=\sum_{\sigma} \exp \left(\frac{1}{2} \beta N_{T}^{-\tau} \sum_{i, j=1}^{N_{T}} \sum_{\mu=1}^{N_{B}} \xi_{i}^{\mu} \xi_{j}^{\mu} \sigma_{i} \sigma_{j}\right) \\
& \bar{f}=-\lim _{N_{T} \rightarrow \infty} \frac{1}{\beta N_{T}} \overline{\log Z_{N_{T}}(\beta, \xi)},
\end{aligned}
$$

where $\cdots$ denotes averaging over the randomly generated $\left\{\xi_{i}^{\mu}\right\}$. If we use the replica identity $\overline{\log Z}=\lim _{n \rightarrow 0} n^{-1} \log \overline{Z^{n}}$, and separate the contributions from the $K$ condensed patterns from those of the $\alpha N_{T}-K$ non-condensed ones we get

$$
\begin{aligned}
\bar{f}= & -\lim _{N_{T} \rightarrow \infty} \lim _{n \rightarrow 0} \frac{1}{\beta n N_{T}} \log \sum_{\sigma^{1}, \cdots, \sigma^{n}} \exp \left(\frac{1}{2} \beta N_{T}^{-\tau} \sum_{i, j=1}^{N_{T}} \sum_{\mu=1}^{N_{B}} \sum_{\alpha=1}^{n} \xi_{i}^{\mu} \xi_{j}^{\mu} \sigma_{i}^{\alpha} \sigma_{j}^{\alpha}\right) \\
= & -\frac{1}{\beta} \log 2-\lim _{N_{T} \rightarrow \infty} \lim _{n \rightarrow 0} \frac{1}{\beta n N_{T}} \log \left(\exp \left(\frac{1}{2} \beta N_{T}^{-\tau} \sum_{\mu=1}^{K} \sum_{\alpha=1}^{n}\left(\sum_{i=1}^{N_{T}} \xi_{i}^{\mu} \sigma_{i}^{\alpha}\right)^{2}\right)\right. \\
& \left.\times \exp \left(\frac{1}{2} \beta N_{T}^{-\tau} \sum_{\mu>K}^{N_{B}} \sum_{\alpha=1}^{n}\left(\sum_{i=1}^{N_{T}} \xi_{i}^{\mu} \sigma_{i}^{\alpha}\right)^{2}\right)\right\rangle_{\sigma^{1}, \ldots, \sigma^{n}} .
\end{aligned}
$$

We compute the non-condensed contributions first, using the standard tool of Gaussian linearization, and the usual short-hands $\mathrm{D} z=(2 \pi)^{-1 / 2} \mathrm{e}^{-z^{2} / 2} \mathrm{~d} z$ and $\mathrm{D} z=\prod_{\alpha=1}^{n} \mathrm{D} z_{\alpha}$ :

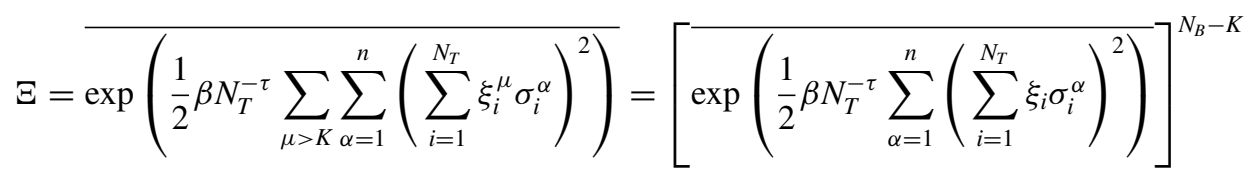

$$
\begin{aligned}
& =\left[\int \mathrm{D} z \exp \left(\sqrt{\beta} N_{T}^{-\tau / 2} \sum_{\alpha=1}^{n} z_{\alpha} \sum_{i=1}^{N_{T}} \xi_{i} \sigma_{i}^{\alpha}\right)\right]^{N_{B}-K} \\
& =\left[\int \mathrm{D} z \prod_{i=1}^{N_{T}}\left(1-c N_{T}^{-\gamma}+c N_{T}^{-\gamma} \cosh \left(\sqrt{\beta} N_{T}^{-\tau / 2} \sum_{\alpha=1}^{n} \sigma_{i}^{\alpha} z_{\alpha}\right)\right)\right]^{N_{B}-K}
\end{aligned}
$$




$$
\begin{aligned}
& =\left[\int \mathrm{D} z \prod_{i=1}^{N_{T}}\left[1+\frac{1}{2} \beta c N_{T}^{-\gamma-\tau}\left(\sum_{\alpha=1}^{n} \sigma_{i}^{\alpha} z_{\alpha}\right)^{2}+O\left(N_{T}^{-2 \tau-\gamma}\right)\right]\right]^{N_{B}-K} \\
& =\left[\int \mathrm{D} z \exp \left(\frac{1}{2} \beta c N_{T}^{-\gamma-\tau} \sum_{\alpha, \beta=1}^{n} z_{\alpha} z_{\beta} \sum_{i=1}^{N_{T}} \sigma_{i}^{\alpha} \sigma_{i}^{\beta}+O\left(N_{T}^{1-2 \tau-\gamma}\right)\right)\right]^{N_{B}-K} .
\end{aligned}
$$

Now it is evident, as in our earlier calculations, that the correct scaling for large $N_{T}$ requires choosing $\tau=1-\gamma$. For the correction term in the exponent this gives $O\left(N_{T}^{1-2 \tau-\gamma}\right)=$ $O\left(N_{T}^{\gamma-1}\right)$, which is indeed vanishing since $\gamma<1$. We now arrive at

$$
\Xi=\exp \left\{\left(N_{B}-K\right) \log \int \mathrm{D} z \exp \left(\frac{1}{2} \beta c N_{T}^{-1} \sum_{\alpha, \beta=1}^{n} z_{\alpha} z_{\beta} \sum_{i=1}^{N_{T}} \sigma_{i}^{\alpha} \sigma_{i}^{\beta}+O\left(N_{T}^{\gamma-1}\right)\right)\right\} .
$$

We next introduce $n^{2}$ parameters $\left\{q_{\alpha \beta}\right\}$ and their conjugates $\left\{\hat{q}_{\alpha \beta}\right\}$, by inserting partitions of unity:

$$
\begin{aligned}
1 & =\prod_{\alpha \beta} \int \mathrm{d} q_{\alpha \beta} \delta\left(q_{\alpha \beta}-\frac{1}{N_{T}} \sum_{i=1}^{N_{T}} \sigma_{i}^{\alpha} \sigma_{i}^{\beta}\right) \\
& =\int\left[\prod_{\alpha \beta} \frac{\mathrm{d} q_{\alpha \beta} \mathrm{d} \hat{q}_{\alpha \beta}}{2 \pi / N_{T}}\right] \exp \left(\mathrm{i} N_{T} \sum_{\alpha, \beta} \hat{q}_{\alpha \beta}\left(q_{\alpha \beta}-\frac{1}{N_{T}} \sum_{i} \sigma_{i}^{\alpha} \sigma_{i}^{\beta}\right)\right) .
\end{aligned}
$$

Substituting (B.6) into (B.5) gives the contribution to the partition function of non-condensed patterns:

$$
\begin{aligned}
\Xi=\int\left[\prod_{\alpha \beta} \mathrm{d} q_{\alpha \beta} \mathrm{d} \hat{q}_{\alpha \beta}\right] \\
\quad \times \exp \left(\mathrm{i} N_{T} \sum_{\alpha, \beta} \hat{q}_{\alpha \beta} q_{\alpha \beta}+\left(N_{B}-K\right) \log \int \mathrm{D} z \mathrm{e}^{\frac{1}{2} \beta c \sum_{\alpha, \beta=1}^{n} z_{\alpha} q_{\alpha \beta} z_{\beta}}+O\left(N_{T}^{\gamma}\right)\right) \\
\quad \times \exp \left(-\mathrm{i} \sum_{i} \sum_{\alpha, \beta} \sigma_{i}^{\alpha} \hat{q}_{\alpha \beta} \sigma_{i}^{\beta}\right) .
\end{aligned}
$$

The contribution from condensed pattern, see (B.3), is

$$
\exp \left(\frac{1}{2} \beta N_{T}^{\gamma-1} \sum_{\mu \leqslant K} \sum_{\alpha=1}^{n}\left(\sum_{i=1}^{N_{T}} \xi_{i}^{\mu} \sigma_{i}^{\alpha}\right)^{2}\right)=\int \mathrm{D} \boldsymbol{m} \exp \left(\sqrt{\beta} N_{T}^{(\gamma-1) / 2} \sum_{\mu \leqslant K} \sum_{\alpha=1}^{n} \sum_{i=1}^{N_{T}} \xi_{i}^{\mu} \sigma_{i}^{\alpha} m_{\alpha}^{\mu}\right),
$$

with $\boldsymbol{m}=\left\{m_{\alpha}^{\mu}\right\} \in \mathbb{R}^{n K}$. If we rescale $m_{\alpha}^{\mu} \rightarrow c \sqrt{\beta} N_{T}^{(1-\gamma) / 2} m_{\alpha}^{\mu}$ this becomes

$$
\left(c^{2} \beta N_{T}^{1-\gamma}\right)^{n K / 2} \int \mathrm{d} \boldsymbol{m} \exp \left(-\frac{1}{2} \beta c^{2} N_{T}^{1-\gamma} \boldsymbol{m}^{2}+\beta c \sum_{\mu \leqslant K} \sum_{\alpha=1}^{n} \sum_{i=1}^{N_{T}} \xi_{i}^{\mu} \sigma_{i}^{\alpha} m_{\alpha}^{\mu}\right) .
$$


Inserting (B.7), (B.9) into (B.3) gives the following expression for the free energy per spin:

$$
\begin{aligned}
\bar{f}= & -\frac{1}{\beta} \log 2-\lim _{N_{T} \rightarrow \infty}\left\{\frac{K / 2}{\beta N_{T}} \log \left(c^{2} \beta N_{T}^{1-\gamma}\right)+\lim _{n \rightarrow 0} \frac{1}{\beta n N_{T}} \log \int \mathrm{d} \boldsymbol{m}\left[\prod_{\alpha \beta} \mathrm{d} q_{\alpha \beta} \mathrm{d} \hat{q}_{\alpha \beta}\right]\right. \\
& \times \exp \left(N_{T}\left[\mathrm{i} \sum_{\alpha, \beta} \hat{q}_{\alpha \beta} q_{\alpha \beta}+\frac{N_{B}-K}{N_{T}} \log \int \mathrm{D} z \mathrm{e}^{\frac{1}{2} \beta c \sum_{\alpha, \beta=1}^{n} z_{\alpha} q_{\alpha \beta} z_{\beta}}-\frac{1}{2} \beta c^{2} N_{T}^{-\gamma} \boldsymbol{m}^{2}\right]\right) \\
& \left.\times \prod_{i=1}^{N_{T}}\left\langle\exp \left(\beta c \sum_{\mu \leqslant K} \sum_{\alpha=1}^{n} \xi_{i}^{\mu} \sigma_{i}^{\alpha} m_{\alpha}^{\mu}-\mathrm{i} \sum_{\alpha, \beta} \sigma_{i}^{\alpha} \hat{q}_{\alpha \beta} \sigma_{i}^{\beta}\right)\right\rangle_{\sigma_{i}^{1}, \ldots, \sigma_{i}^{n}}\right\} .
\end{aligned}
$$

The number of order parameters being integrated over is of order $K$, so corrections to the saddle-point contribution will be of order $O(K \log N / N)$. To proceed via steepest descent we must therefore impose $K \ll N_{T} / \log N_{T}$. Since also the energy term $N_{T}^{-\gamma} \sum_{\mu \leqslant K} \boldsymbol{m}^{2}$ should be of order 1 , as well as the individual components of $\boldsymbol{m}$, the only natural choice is $K=O\left(N^{\gamma}\right)$. Under this scaling condition we then find

$$
\bar{f}=-\frac{1}{\beta} \log 2-\lim _{K \rightarrow \infty} \lim _{n \rightarrow 0} \frac{1}{\beta n} \operatorname{extr}_{\boldsymbol{m}, q, \hat{q}} \hat{f}(\boldsymbol{m},\{q, \hat{q}\})
$$

with

$\hat{f}(\boldsymbol{m},\{q, \hat{q}\})=\mathrm{i} \sum_{\alpha, \beta} \hat{q}_{\alpha \beta} q_{\alpha \beta}+\alpha \log \int \mathrm{D} z \exp \left(\frac{1}{2} \beta c \sum_{\alpha, \beta=1}^{n} z_{\alpha} q_{\alpha \beta} z_{\beta}\right)-\frac{\beta c^{2}}{2 N_{T}^{\gamma}} \sum_{\alpha=1}^{n} \sum_{\mu \leqslant K}\left(m_{\alpha}^{\mu}\right)^{2}$

$$
+\left\langle\log \left\langle\exp \left(\beta c \sum_{\mu \leqslant K} \sum_{\alpha=1}^{n} \xi^{\mu} \sigma^{\alpha} m_{\alpha}^{\mu}-\mathrm{i} \sum_{\alpha, \beta} \sigma^{\alpha} \hat{q}_{\alpha \beta} \sigma^{\beta}\right)\right\rangle_{\sigma^{1}, \ldots, \sigma^{n}}\right\rangle .
$$

Now we can use the replica symmetry ansatz, and demand that the relevant saddle-point is of the form

$m_{\alpha}^{\mu}=m^{\mu}, \quad q_{\alpha \beta}=\delta_{\alpha \beta}+q\left(1-\delta_{\alpha \beta}\right), \quad \hat{q}_{\alpha \beta}=\frac{\mathrm{i} \alpha(\beta c)^{2}}{2}\left[R \delta_{\alpha \beta}+r\left(1-\delta_{\alpha \beta}\right)\right]$.

From now on we will denote $\boldsymbol{m}=\left(m^{1}, \ldots, m^{K}\right)$ and $\boldsymbol{\xi}=\left(\xi^{1}, \ldots, \xi^{K}\right)$. After some simple algebra we can take the limit $n \rightarrow 0$, and find that our free energy simplifies to

$$
\beta \bar{f}_{\mathrm{RS}}=\lim _{N_{T} \rightarrow \infty} \operatorname{extr}_{m, q, r} \hat{f}_{\mathrm{RS}}(\boldsymbol{m}, q, r)
$$

with

$$
\begin{aligned}
\hat{f}_{\mathrm{RS}}(\boldsymbol{m}, q, r)= & -\log 2+\frac{1}{2} \alpha r(\beta c)^{2}(1-q)+\frac{\beta c^{2}}{2 N_{T}^{\gamma}} \boldsymbol{m}^{2} \\
& -\frac{\alpha}{2}\left(\frac{\beta c q}{1-\beta c(1-q)}-\log [1-\beta c(1-q)]\right) \\
& -\left\langle\int \mathrm{D} z \log \cosh [\beta c(\boldsymbol{m} \cdot \boldsymbol{\xi}+z \sqrt{\alpha r})]\right\rangle_{\xi}
\end{aligned}
$$




\section{References}

[1] Parisi G 1990 Proc. Natl Acad. Sci. USA 87 429-33

[2] Barra A and Agliari E 2010 J. Stat. Mech. P07004

[3] Agliari E, Asti L, Barra A and Ferrucci L 2012 Phys. Rev. E 85051909

[4] Agliari E, Barra A, Guerra F and Moauro F 2011 J. Theor. Biol. 287 48-63

[5] Mora T, Walczak A M, Bialek W and Callan C G 2010 Proc. Natl Acad. Sci. USA $1075405-10$

[6] Košmrlj A, Chakraborty A K, Kardar M and Shakhnovich E I 2009 Phys. Rev. Lett. 103068103

[7] Košmrlj A, Jha A K, Huseby E S, Kardar M and Chakraborty A K 2008 Proc. Natl Acad. Sci. USA 105 16671-6

[8] Chakraborty A K and Košmrlj A 2011 Annu. Rev. Phys. Chem. 61 283-303

[9] Schmidtchen H, Thüne M and Behn U 2012 Phys. Rev. E 86011930

[10] Uezu T, Kadano C, Hatchett J P L and Coolen A C C 2006 Prog. Theor. Phys. 161 385-8

[11] Perelson A S and Weisbuch G 1997 Rev. Mod. Phys. 69 1219-67

[12] De Boer R J, Perelson A S and Kevrekidis I G 1993 Bull. Math. Biol. 55 745-80

[13] Nesterenko V G 1988 Theoretical Immunology ed A S Perelson (New York: Addison-Wesley)

[14] Farmer J D, Packard N H and Perelson A S 1986 Physica D 22 187-204

[15] Brede M and Behn U 2001 Phys. Rev. E 64011908

[16] Brede M and Behn U 2003 Phys. Rev. E 67031920

[17] Madi A et al 2011 Chaos 21016109

[18] Madi A et al 2011 PLoS One 6 e17445

[19] Quintana F J and Cohen I R 2011 Trends Immunol. 32 89-95

[20] Weisbuch G 1990 J. Theor. Biol. 143507

[21] Pan K and Deem M W 2011 Phys. Biol. 8055006

[22] Deem M W and Lee H Y 2003 Phys. Rev. Lett. 91068101

[23] Vertosick F T and Kelly R H 1989 Immunology 661

[24] Merbl Y et al 2009 PLoS One 4 e6053

[25] Madi A et al 2009 Proc. Natl Acad. Sci. USA 10614484

[26] Medzhitov R 2007 Nature 44928

[27] Medzhitov R 2009 Immunity 30776

[28] Bersini H and Varela F 1991 Lect. Notes Comput. Sci. 496343

[29] Hoffmann G W 1986 J. Theor. Biol. 12233

[30] Agliari E, Barra A, Galluzzi A, Guerra F and Moauro F 2012 Phys. Rev. Lett. 109268101

[31] Agliari E, Barra A, Bartolucci S, Galluzzi A, Guerra F and Moauro F 2013 Phys. Rev. E 87042701

[32] Barra A, Genovese G and Guerra F 2010 J. Stat. Phys. $140784-96$

[33] Barra A, Bernacchia A, Contucci P and Santucci E 2012 Neural Netw. 34 1-9

[34] Barkai E, Kanter I and Sompolinsky H 1990 Phys. Rev. A 41 1843-54

[35] Coolen A C C 2001 Handbook of Biological Physics vol 4 ed F Moss and S Gielen (Amsterdam: Elsevier) pp 531-59

[36] Perez-Castillo I, Wemmenhove B, Hatchett J P L, Coolen A C C, Skantzos N S and Nikoletopoulos T 2004 J. Phys. A: Math. Gen. 37 8789-99

[37] Skantzos N S and Coolen A C C 2000 J. Phys. A: Math. Gen. 33 5785-807

[38] Sompolinsky H 1986 Phys. Rev. A 34 2571-4

[39] Wemmenhove B and Coolen A C C 2003 J. Phys. A: Math. Gen. 36 9617-33

[40] Janeway C, Travers P, Walport M and Shlomchik M 2005 Immunobiology (New York: Garland Science Publishing)

[41] Abbas A K, Lichtman A H and Pillai S 2012 Cellular and Molecular Immunology (Philadelphia, PA: Elsevier)

[42] Barra A and Agliari E 2010 Physica A 389 5903-11

[43] Theze J (ed) 1999 The Cytokine Network and Immune Functions (Oxford: Oxford University Press)

[44] Kuchroo V, Sarvetnick N, Hafler D and Nicholson L (ed) 2002 Cytokine and Autoimmune Diseases (Totowa: Humana Press)

[45] Goodnow C C 1992 Annu. Rev. Immunol. 10 489-518

[46] Goodnow C C 2005 Nature 435590

[47] Goodnow C C, Vinuesa C G, Randall K L, Mackay F and Brink R 2010 Nature Immunol. 8681

[48] Schwartz R H 2005 Nature Immunol. 4327

[49] Derrida B, Gardner E and Zippelius A 1987 Europhys. Lett. 4 167-73

[50] Wemmenhove B, Skantzos N S and Coolen A C C 2004 J. Phys. A: Math. Gen. 37 7653-70

[51] Amit D J 1992 Modeling Brain Function: The World of Attractor Neural Networks (Cambridge: Cambridge University Press) 
[52] Amit D J, Gutfreund H and Sompolinsky H 1987 Phys. Rev. A 35 2293-303

[53] Newman M E J 2002 Phys. Rev. E 66016128

[54] Allard A, Noël P-A, Dubé L J and Pourbohloul B 2009 Phys. Rev. E 79036113

[55] Barra A 2008 J. Stat. Phys. 132 787-809

[56] Perez-Vicente C J and Coolen A C C 2008 J. Phys. A: Math. Theor. 41255003 